\title{
Impact of Polypharmacy on Candidate Biomarker miRNomes for the Diagnosis of Fibromyalgia and Myalgic Encephalomyelitis/Chronic Fatigue Syndrome: Striking back on Treatments
}

\author{
Eloy Almenar-Pérez ${ }^{1}$, Teresa Sánchez-Fito ${ }^{2}$, Tamara Ovejero ${ }^{3}$, Lubov Nathanson ${ }^{4}$ and Elisa \\ Oltra $^{5, *}$ \\ 1 Escuela de Doctorado, Universidad Católica de Valencia San Vicente Mártir, 46001 Valencia, Spain; \\ eloy.almenar@ucv.es \\ 2 Escuela de Doctorado, Universidad Católica de Valencia San Vicente Mártir, 46001 Valencia, Spain; \\ mt.sanchez@ucv.es \\ 3 School of Medicine, Universidad Católica de Valencia San Vicente Mártir, 46001 Valencia, Spain; \\ tamara.ovejero@ucv.es \\ 4 Kiran C Patel College of Osteopathic Medicine, Nova Southeastern University, Ft Lauderdale 33314, USA; \\ Institute for Neuro Immune Medicine, Nova Southeastern University, Ft Lauderdale 33314, USA; \\ lnathanson@nova.edu \\ 5 School of Medicine, Universidad Católica de Valencia San Vicente Mártir, 46001 Valencia, Spain; \\ Unidad mixta CIPF-UCV, Centro de Investigación Príncipe Felipe, 46012 Valencia, Spain \\ * Correspondence: elisa.oltra@ucv.es ; Tel.: +34-963637412
}

\begin{abstract}
Fibromyalgia (FM) and Myalgic Encephalomyelitis/Chronic Fatigue Syndrome (ME/CFS) are diseases of unknown etiology presenting complex and often overlapping symptomatology. Despite promising advances on the study of miRNomes of these diseases, no validated molecular diagnostic biomarker yet exists. Since FM and ME/CFS patient treatments commonly include polypharmacy it is of concern that biomarker miRNAs are masked by drug interactions. Aiming at discriminating between drug-effects and true disease-associated differential miRNA expression, we evaluated the potential impact of commonly prescribed drugs on disease miRNomes, as reported by the literature. By using the web search tools SM2miR, Pharmaco-miR and repoDB, we found a list of commonly prescribed drugs that impact on FM and ME/CFS miRNomes and therefore could be interfering in the process of biomarker discovery. On another end, disease-associated miRNomes may incline patient's response to treatment and toxicity. Here, we explored treatments for diseases in general that could be affected by FM and ME/CFS miRNomes finding a long list of them, including treatments for lymphoma, a type of cancer affecting ME/CFS patients at a higher rate than healthy population. We conclude that FM and ME/CFS miRNomes could help refine pharmacogenomic/pharmacoepigenomic analysis to elevate future personalized medicine and precision medicine programs in the clinic.
\end{abstract}

Keywords: Fibromyalgia (FM); Myalgic Encephalomyelitis/Chronic Fatigue Syndrome (ME/CFS); microRNA; miRNome; pharmacogenomics; pharmacoepigenomics; SM2miR; Pharmaco-miR; repoDB; ME/CFS Common Data Elements (CDEs).

\section{Introduction}

Fibromyalgia (FM) is a debilitating disorder characterized by low pain threshold and muscle tenderness accompanied by bowel abnormalities, sleep disturbances, depressive episodes, cognitive problems and chronic pain [1-4]. Despite commonly comorbid with Myalgic Encephalomyelitis/Chronic Fatigue Syndrome (ME/CFS), a disease also showing a complex clinical pathophysiology [5-11], these syndromes have been classified by the International Classification of 
Diseases, Tenth Revision, Clinical Modification (ICD-10-CM) with separate codes (M79.7 and R53.82 or G93.3 if post-viral, for FM and ME/CFS respectively) [12]. However, disease distinctions remain under debate [5].

Although possibly underestimated, the global prevalence for FM has been set at $2-8 \%$ and at 0.23-0.41 for ME/CFS with predominant ratios of females over males [13-17]. In addition, increasing numbers of patients being affected at early ages [18] highlights the considerable and raising needs for appropriate healthcare programs and the stepping demands for alleviation of associated economic/social burdens.

Post-exertional malaise (PEM) a clinical hallmark of ME/CFS together with additional clinical and biological parameters differing between these two diseases [19-24] seem to support a distinct underlying pathophysiology and possibly etiology for FM and ME/CFS. Aimed at clarifying this diagnostic conflict through an improved understanding of the biology of disease onset and evolution, some research groups, ours included, have set searches to identify molecular biomarkers of these illnesses [25].

MicroRNAs or miRs constitute attractive candidates for the diagnosis of FM and ME/CFS as they have been found to associate with disease state of other complex chronic diseases [26,27] and may even be used to measure disease stage and response to treatments [28]. In their mature form (20-22 $\mathrm{nts})$, they epigenetically control gene expression by directing particular sets of mRNAs presenting partial complementation, often, in their 3'UTR region, to degradation [29]. Other regulatory mechanisms have also been linked to the activity of these small molecules [30].

In addition to their biomarker value, miRNAs could potentially be targeted by small drugs, either directly through the binding of chemical compounds to particular grooves or pockets of their secondary structures, in their mature or precursor forms, as isolated or complexed molecules; or indirectly by interfering with proteins involved in their biogenesis or recycling, including regulation of transcription factors driving miRNA synthesis [31-34]. Therefore, directional FM and ME/CFS treatments based on miRNA targeting strategies are envisioned as potential curative therapies by themselves or as co-adjuvants in a near future.

MiRNA capacity to sense and respond to environmental cues [35-37], however, makes the establishment of correlations between particular disease estates and miRNA profile changes challenging. To minimize potential environmental confounding factors healthy participants are often population matched by sex, age; and quite frequently by BMI (body mass index) with the participating patient group. Careful selection of participants and proper study design are key factors in identifying miRNA disease-associated profiles (disease miRNomes), as miRNA levels also change in response to hormone challenges, during aging and metabolic states [38-41], including the postprandial estate [42]. In the context of FM and ME/CFS, since miRNomes change with exercise [43], inclusion of sedentary control groups would be desirable.

Current treatments of both, FM and ME/CFS, diseases are symptom palliative only [44-48]. Due to multi-symptomatology, patient prescriptions frequently involve polypharmacy which may significantly impact downstream molecular analysis of disease. With this perspective, a recent joint initiative worked out by the NINDS (National Institute of Neurological Disorders and Stroke) at the NIH (National Institute of Health) in Bethesda, MD (USA) and other federal agencies has made available case report forms (CRFs) and guidelines to register drug use in ME/CFS studies [49]. The ME/CFS Common Data Elements initiative or CDE Project aimed at standardizing clinical relevant variables for the study of ME/CFS covers various areas organized by domains and sub-domains. Information is publicly available at: https://www.commondataelements.ninds.nih.gov/MECFS.aspx\#tab=Data Standards [49].

A recently observed feature of miRNAs is their role at determining drug efficacy [50,51]. The traditional field of pharmacogenomics dealing at how individual genomic features, including SNPs (single nucleotide polymorphisms) and CNV (copy number variants), influences patient's response to drug-based treatments and sensitivity to toxic effects is becoming complemented by individual epigenetic profiles including alternative splicing events and miRNomes (pharmacoepigenomics) with the aim of elevating predictions of most effective and safest options towards improved personalized treatments in the clinic [52-55]. In addition to epigenetic regulation of drug targets, 
regulation of genes related to drug absorption, distribution, metabolism, and excretion (ADME) may translate into significant inter-individual differences to drug response [56]. In this context it should be of relevance to take into account patient's FM or/and ME/CFS condition when standardized treatments for diseases other than FM and ME/CFS are at need. In particular, FM and ME/CFS associated miRNA profiles might promote drug efficacy or inhibit drug function when compared to non-FM and non-ME/CFS patients and consequently impact or influence FM and ME/CFS patient's response to pharmacological treatments or sensitivity to adverse reactions. Interestingly, and in line with this, FM and ME/CFS patients report suffering from multiple chemical sensitivity [57].

In this paper we have interrogated the potential impact of commonly prescribed drugs to treat FM and ME/CFS on miRNA profiles in an effort to discern between miRNAs potentially linked to disease from those that might be consequence of drug intake. On another side, we have evaluated miRNA-target genes-drug interactions of differentially expressed (DE) miRNAs in FM and ME/CFS as an approach to determine the ability or predisposition of these patients to respond to common clinical treatments for disease in general, including diseases other than FM and ME/CFS which may appear comorbid at some point of FM and ME/CFS patients' lives.

\section{Materials and Methods}

\subsection{Study search}

To locate experimental work aimed at studying miRNA profiles in FM or ME/CFS, a bibliographic search following the Preferred Reporting Items for Systematic Reviews and MetaAnalyses criteria (PRISMA)[58] was performed using Pubmed and Web of Science databases [59,60] up to January 2019. The search terms used in "all fields" included: "fibromyalgia" AND "microRNA" OR "miR" on one side, and "chronic fatigue syndrome" AND "CFS" in combination with "microRNA" OR "miR" in another. The use of the following terms: "myalgic encephalomyelitis" to describe the disease did not yield any additional experimental publications in the field. The trial Pubmed Labs tool, including article snippets and other improvements was also used in the search [61]. Manual curation to filter out non-experimental or unrelated hits was applied.

For compounds commonly prescribed to treat symptoms in FM and ME/CFS a search in Cochrane library and Pubmed databases [59,62] was performed using as search terms either "fibromyalgia systematic review" AND "drug" or "chronic fatigue syndrome" AND "CFS systematic review" AND "drug" or "myalgic encephalomyelitis systematic review" AND "drug". Most recently updated reviews were adopted as reference manuscripts.

\subsection{Identification of miRNA-drug-disease interactions}

Features of miRNAs and drugs understudy, International Union of Pure and Applied Chemistry (IUPAC) names included, were found in miRBase and Drugbank databases, respectively [63,64]. FM and ME/CFS miRNomes were evaluated for miRNA-drug interactions using either SM2miR or Pharmaco-miR web search tools [65,50]. To find potential miRNomes derived from patient polypharmacy, SM2miR output was filtered using as criteria "drugs commonly prescribed to treat FM and ME/CFS symptoms", as described in the previous 2.1. section. Treatments to disease to which FM and ME/CFS patients may respond differently than non-FM non-ME/CFS population were spotted by searching the repoDB database [66] with the Pharmaco-miR drug hits obtained with FM or ME/CFS miRNome searches. 


\section{Results}

\section{1. miRNomes associating with the studied diseases}

\subsection{1. miRNomes of FM}

By reviewing the literature, as described in Methods, we found five studies reporting differential expression (DE) of particular miRNAs in FM patients with respect to healthy population using multiplex approaches, either microarrays or RT-qPCR panels (Table S1 \&Table 1). One of them measured miRNA levels in cerebrospinal fluid (CSF)[67], while the rest evaluated them in blood fractions [68-71]; two used white blood cells [69,71] and two analyzed serum [68,70].

According to these reports, a total of $85 \mathrm{FM}$ patients and 86 healthy participants have been screened for differential miRNA expression finding little coincidences (only 9 miRNAs reported by more than one study) (Table 1, miRNAs in bold) even within the same blood fraction type and although authors report use of common diagnostic criteria (ACR 1990). Gene Ontology (GO) analysis, however, more commonly showed metabolic and neural pathways associating to DE miRNAs, indicating common cellular pathways affected by different FM miRNomes.

Table 1. Summary of studies evaluating FM miRNomes by multiplex approaches.

\begin{tabular}{|c|c|c|c|c|c|c|c|c|}
\hline $\begin{array}{l}\text { Source of } \\
\text { RNA }\end{array}$ & $\begin{array}{c}\text { Diagnostic } \\
\text { criteria } \\
\text { /Clinical } \\
\text { parameters } \\
\end{array}$ & Cohorts & $\begin{array}{l}\text { Technical } \\
\text { approach }\end{array}$ & Over-expressed microRNAs & $\begin{array}{l}\text { Under-expressed } \\
\text { microRNAs }\end{array}$ & $\begin{array}{l}\text { RT-qPCR } \\
\text { validated } \\
\text { miRNAs }\end{array}$ & $\begin{array}{l}\text { GO terms mainly } \\
\text { affected }\end{array}$ & References \\
\hline $\begin{array}{l}\text { Cerebrospinal } \\
\text { fluid (CSF) }\end{array}$ & $\begin{array}{l}\text { ACR 1990, } \\
\text { FIQ \& MFI- } \\
20^{*}\end{array}$ & $\begin{array}{l}10 \mathrm{FM} \\
8 \mathrm{HC}\end{array}$ & $\begin{array}{l}\text { microRNA Ready- } \\
\text { to-Use PCR } \\
\text { microchip (Exiqon, } \\
\text { Denmark Cat No } \\
\text { 203608) }\end{array}$ & & $\begin{array}{l}\text { miR-21-5p, miR-145- } \\
\text { 5p, miR-29a-3p, miR- } \\
\text { 99b-5p, miR-125b-5p, } \\
\text { miR-23a-3p, miR-23b- } \\
\text { 3p, miR-195-5p, miR- } \\
\underline{\text { 223-3p }}\end{array}$ & N/A & $\begin{array}{l}\text { Glial and Neuronal } \\
\text { response, Insulin-like } \\
\text { growth factor pathway, } \\
\text { Alzheimer and } \\
\text { Parkinson, } \\
\text { Autoimmunity and } \\
\text { Energy metabolism }\end{array}$ & $\begin{array}{l}\text { Bjersing et } \\
\text { al, 2013[67] }\end{array}$ \\
\hline Serum & $\begin{array}{l}\text { ACR 1990, } \\
\text { FIQ \& MFI- } \\
20^{*}\end{array}$ & $\begin{array}{l}20 \mathrm{FM} \\
20 \mathrm{HC}\end{array}$ & $\begin{array}{l}\text { microRNA Ready- } \\
\text { to-Use PCR } \\
\text { microchip } \\
\text { (Exiqon, Denmark } \\
\text { Cat No 203608) }\end{array}$ & $\underline{\operatorname{miR}-320 a}$ & $\begin{array}{l}\text { miR-103a-3p, miR- } \\
\text { 107, let-7a-5p, mir- } \\
\text { 30b-5p, miR-151a-5p, } \\
\text { miR-142-3p, miR- } \\
\text { 374b-5p. }\end{array}$ & N/A & $\begin{array}{l}\text { Neuronal regeneration, } \\
\text { Opioid tolerance, } \\
\text { Dopamine } \\
\text { neurotransmitter } \\
\text { receptor activity, cell } \\
\text { division, stress response, } \\
\text { energy metabolism, lipid } \\
\text { metabolism, Alzheimer }\end{array}$ & $\begin{array}{l}\text { Bjersing et } \\
\text { al, 2014[68] }\end{array}$ \\
\hline PBMCs & $\begin{array}{l}\text { ACR 1990, } \\
\text { FIQ \& MFI- } \\
20^{*}\end{array}$ & $\begin{array}{l}11 \mathrm{FM} \\
10 \mathrm{HC}\end{array}$ & $\begin{array}{l}\text { 3D-Gene Human } \\
\text { miRNA Oligo chips } \\
\text { (version 16.0; Toray } \\
\text { Industries) }\end{array}$ & & $\begin{array}{l}\text { miR-223-3p, miR- } \\
451 a, \text { miR-338-3p, } \\
\text { miR-143-3p, miR-145- } \\
\text { 5p, miR-21-5p }\end{array}$ & $\begin{array}{l}\text { miR-223-3p, } \\
\text { miR-451a, miR- } \\
\text { 338-3p, miR-143- } \\
\text { 3p, miR-145-5p }\end{array}$ & $\begin{array}{l}\text { Nervous system, } \\
\text { Inflammation, Diabetes, } \\
\text { Major depressive } \\
\text { disorder, }\end{array}$ & $\begin{array}{l}\text { Cerdá- } \\
\text { Olmedo et } \\
\text { al, 2015[69] }\end{array}$ \\
\hline Serum & $\begin{array}{c}\text { ACR } \\
\text { 1990/2010, } \\
\text { FIQ, FAS, } \\
\text { HAQ \& } \\
\text { ZSAS/ZSD } \\
\text { S }^{*}\end{array}$ & $\begin{array}{l}14 \mathrm{FM} \\
14 \mathrm{HC}\end{array}$ & $\begin{array}{l}\text { Serum/Plasma } \\
\text { Focus miRNA PCR } \\
\text { Panel I+II (96-wells } \\
\text { Exiqon) }\end{array}$ & $\begin{array}{l}\text { Pooled Sera: } \text { miR-10a-5p, miR-320b, } \\
\text { miR-424-5p }\end{array}$ & $\begin{array}{l}\text { Pooled Sera: miR-20a- } \\
\text { 3p, miR-139-5p } \\
\text { Individual Sera: miR- } \\
\text { 23a-3p, miR-1, miR- } \\
\text { 133a, miR-346, miR- } \\
\text { 139-5p, miR-320b }\end{array}$ & N/A & $\begin{array}{l}\text { Brain development, } \\
\text { Immune response, } \\
\text { Osteogenesis, Myoblast } \\
\text { differentiation, Autism, } \\
\text { Epilepsy, Cellular } \\
\text { proliferation and } \\
\text { differentiation, Muscular } \\
\text { athrophy, Complex } \\
\text { regional pain syndrome, } \\
\text { among other }\end{array}$ & $\begin{array}{l}\text { Masotti et } \\
\text { al, 2016[70] }\end{array}$ \\
\hline $\begin{array}{l}\text { White blood } \\
\text { cell } \\
\text { (WBC) }\end{array}$ & $\begin{array}{c}\text { ACR 1990, } \\
\text { FIQ, NPSI- } \\
\text { G, } \\
\text { GCPS \& } \\
\text { ADS* }\end{array}$ & $\begin{array}{l}30 \mathrm{FM} \\
34 \mathrm{HC}\end{array}$ & $\begin{array}{l}\text { miRCURY LNA } \\
\text { miRNA array } \\
\text { (Exiqon, Vedbaek, } \\
\text { version 19.0, with } \\
2042 \text { analyzed } \\
\text { microRNAs) }\end{array}$ & $\begin{array}{l}\text { miR-136-5p, miR-4306, miR-744-5p, } \\
\text { miR-4301, miR-151a-3p, miR-584-5p, } \\
\text { miR-4288, miR-221-3p, miR-151a-5p, } \\
\text { miR-199a-5p, miR-126-3p, miR-126-5p, } \\
\text { miR-130a-3p, miR-146a-5p, miR-125a- } \\
\text { 5p, miR-4429, miR-320b miR-320a, } \\
\text { miR-320c, miR-17-3p, miR-423-3p, } \\
\text { miR-425-5p, miR-4291, miR-652-3p, } \\
\text { miR-103b-3p, miR-199a-3p, miR-335- } \\
\text { 5p, miR-331-3p, miR-339-5p, miR-92a- } \\
\text { 3p, let-7b-5p, miR-222-3p, miR-33a, } \\
\text { let-7i-5p, miR-185-5p, miR-22-3p }{ }_{\llcorner} \text {miR- } \\
\text { 148b-3p, miR-103a-3p, let-7d-5p, miR- } \\
\text { 4289, miR-107, miR-30d-5p, miR-301a- } \\
\text { 3p, miR-374c-5p, miR-17-5p, miR-18b- } \\
\text { 5p, miR-1 }\end{array}$ & $\begin{array}{l}\text { miR-4639-3p, miR- } \\
\text { 3685, miR-943, miR- } \\
\text { 877-3p }\end{array}$ & $\begin{array}{l}\text { miR-199a, miR- } \\
\text { 151, miR-103, } \\
\text { Let-7d, miR-146a }\end{array}$ & $\begin{array}{l}\text { Cell proliferation, } \\
\text { differentiation, brain } \\
\text { development, Opioid } \\
\text { Tolerance }\end{array}$ & $\begin{array}{l}\text { Leinders et } \\
\text { al, 2016[1] }\end{array}$ \\
\hline
\end{tabular}


*ACR: American College of Rheumatology 1990/2010 criteria; FIQ; Fibromyalgia Impact Questionnaire; MFI-20: Multidimensional Fatigue Inventory; FAS: Fibromyalgia Assessment Status; HAQ: Health Assessment Questionnaire; ZSAS, ZSDS: Zung Self-Rating Anxiety and Zung SelfRating Depression Scale; NPSI-G: Neuropathic Pain Symptom Inventory; GCPS: Graded Chronic Pain Scale; ADS: Allgemeine Depressions-Skala. Bolded miRs correspond to miRs DE according to more than one FM study. Underlined miRs correspond to miRs DE in FM and ME/CFS studies.

\subsection{2. miRNomes of ME/CFS}

A similar bibliographic search to the one performed in FM (section 3.1.1. of this manuscript) yielded, after filtering out unrelated, gene-focussed studies, only three studies showing DE of miRNAs in ME/CFS at basal levels, yet, they included a similar total number of patients and controls (83 and 98, respectively) (Table 2) [72-74]. It should be noted that an additional multiplex miRNA study evaluating DE of miRNAs in ME/CFS upon exercise challenge was excluded on the basis of reporting no basal disease miRNomes [75]. Again, as in FM studies, little overlap of DE miRNAs could be found across ME/CFS studies (only 4 miRNAs were reported by more than one ME/CFS study, bolded miRNAs in Table 2). In this case, this could be somehow expected as blood fractions and diagnostic criteria varied across studies. In fact, only the most recent study by Petty et al., included the more restrictive Canadian criteria for patient selection [74]. Nevertheless, once more, a coincidence of mainly affected GO terms was found, pointing at major immune defects in ME/CFS through different miRNomes.

Table 2. Summary of studies evaluating ME/CFS miRNomes by multiplex approaches

\begin{tabular}{|c|c|c|c|c|c|c|c|c|}
\hline $\begin{array}{l}\text { Source of } \\
\text { RNA }\end{array}$ & $\begin{array}{c}\text { Diagnostic } \\
\text { criteria }\end{array}$ & Cohorts & $\begin{array}{l}\text { Technical } \\
\text { approach }\end{array}$ & $\begin{array}{l}\text { Over-expressed } \\
\text { microRNAs }\end{array}$ & $\begin{array}{l}\text { Under-expressed } \\
\text { microRNAs }\end{array}$ & $\begin{array}{l}\text { RT-qPCR } \\
\text { validated } \\
\text { microRNAs } \\
\end{array}$ & $\begin{array}{l}\text { GO terms mainly } \\
\text { affected }\end{array}$ & References \\
\hline $\begin{array}{c}\text { NK \& } \\
\text { CD8+ cells }\end{array}$ & Fukuda & $\begin{array}{c}28 \mathrm{ME} / \mathrm{CFS} \\
28 \mathrm{HC}\end{array}$ & $\begin{array}{l}\text { Analyzed by RT-qPCR } 19 \\
\text { microRNAs: miR-10a miR- } \\
\text { 16,miR-15b,miR-107,miR- } \\
\text { 128b,miR-146a,miR-191,miR- } \\
\text { 21,miR-223, ,miR-17-5p,miR- } \\
\text { 150,miR-103,miR-106b,miR- } \\
\text { 126,miR-142-3p,miR-146- } \\
\text { 5p,miR-152,miR-181,let-7a. }\end{array}$ & & 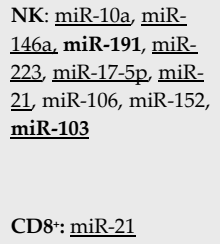 & N/A & $\begin{array}{l}\text { Cytotoxicity of NK } \\
\text { and CD8 } 8^{+} \text {cells, } \\
\text { cytokine expression, } \\
\text { cell proliferation, } \\
\text { apoptosis, } \\
\text { development and } \\
\text { differentiation of } \\
\text { effector CD8 } 8^{+}\end{array}$ & $\begin{array}{l}\text { Brenu et al., } \\
2012^{[72]}\end{array}$ \\
\hline Plasma & Fukuda & $\begin{array}{c}20 \mathrm{ME} / \mathrm{CFS} \\
20 \mathrm{HC}\end{array}$ & $\begin{array}{l}\text { MicroRNA profiling by } \\
\text { HiSeq2000 sequencing } \\
\text { (Illumina HiSeq2000) }\end{array}$ & $\begin{array}{l}\text { miR-548j, miR-548ax, miR- } \\
127-3 p, \text { miR-381-3p, miR- } \\
\underline{331-3 p} \text { miR-136-3p, miR- } \\
\text { 142-5p, miR-493-5p, miR- } \\
\underline{143-3 p} \text {, miR-370, miR-4532 }\end{array}$ & $\begin{array}{l}\text { miR-126, miR-450b- } \\
\text { 5p, miR-641, miR-26a- } \\
\text { 1-3p, miR-3065-3p, } \\
\text { miR-5187-3p, miR-16- } \\
\text { 2-3p, let-7g-3p }\end{array}$ & $\begin{array}{l}\text { miR-127-3p, } \\
\text { miR-142-5p, } \\
\text { miR-143-3p }\end{array}$ & $\begin{array}{l}\text { Autoimmunity, } \mathrm{T} \text { cell } \\
\text { development, } \\
\text { cytokine production, } \\
\text { inflammatory } \\
\text { responses, apoptosis }\end{array}$ & $\begin{array}{l}\text { Brenu et al., } \\
2014^{[73]}\end{array}$ \\
\hline PBMCs & $\begin{array}{l}\text { Fukuda \& } \\
\text { Canadian }\end{array}$ & $\begin{array}{c}35 \mathrm{ME} / \mathrm{CFS} \\
50 \mathrm{HC}\end{array}$ & $\begin{array}{l}\text { Ambion Bioarray microarrays } \\
\text { (version } 1 \text { targeting } 385 \\
\text { miRNA sequences) }\end{array}$ & $\begin{array}{l}\text { let-7b, miR-103, miR-126, } \\
\text { miR-145, miR-151, miR- } \\
\text { 181a, miR-185, miR-191, } \\
\text { miR-197, miR-199a, miR- } \\
\text { 19b, miR-210, miR-22-5p, } \\
\text { miR-24, miR-27a, miR-27b, } \\
\text { miR-30c, miR-30d, miR-320, } \\
\text { miR-324-3p, miR-324-5p, } \\
\text { miR-326, miR-330, miR- } \\
\text { 331-3p, miR-339, miR-422b, } \\
\text { miR-423, miR-92, miR-99b }\end{array}$ & & $\begin{array}{l}\text { miR-99b, } \\
\text { miR-30c, } \\
\text { miR-126, } \\
\text { miR-330-3p }\end{array}$ & $\begin{array}{l}\text { Angiogenesis, } \\
\text { invasion, migration } \\
\text { and proliferation in } \\
\text { dendritic cells, , } \\
\text { proliferative, cytotoxic } \\
\text { and cytokine effector } \\
\text { function in NK cells }\end{array}$ & $\begin{array}{l}\text { Petty, et al., } \\
2016^{174]}\end{array}$ \\
\hline
\end{tabular}

Bolded miRs correspond to miRs DE according to more than one ME/CFS study. Underlined miRs correspond to miRs DE in FM and ME/CFS studies. This Table has been adapted from Almenar-Perez et al., 2019 (Clinical Therapeutics, under review).

Surprisingly as many as 19 miRNAs were found to be commonly reported as DE by FM and ME/CFS studies, the significance of which is unknown at present (miRNAs underlined in Tables 1 \& 2). 


\subsection{Polypharmacy potentially impacting miRNA profiles}

As mentioned above, our general aims included determining drug-miRNA and drug-disease interactions in the context of FM, or ME/CFS miRNomes, for the purpose of identifying potential interference of drugs in miRNA profiling which could bias research outcomes one side, and, on another, determine whether disease miRNA profiles could influence drug response in these patients. This section focuses on selecting drugs commonly prescribed to FM and ME/CFS patients to evaluate the effect that polypharmacy might have on miRNomes of these diseases.

\subsubsection{Polypharmacy in FM}

Based on the recent Cochrane report by Häuser et al, [44] drugs that have been commonly used to treat FM in the clinical practice can be classified into the following six classes: antidepressants, antiepileptics, antipsicotics, cannabinoids, nonsteroidal anti-inflammatory drugs (NSAIDs) and opioids. Rather than analyzing the quality of evidence of clinical trials using these substances, we were interested in assigning the active principle and IUPAC names to the reported compounds, to facilitate our downstream analysis (Table 3). Additional literature supporting the use of compounds for each of the six classes described by Häuser et al., to treat FM patients is provided in Table 3 [7693].

Table 3. Classification of drugs commonly prescribed to FM patients

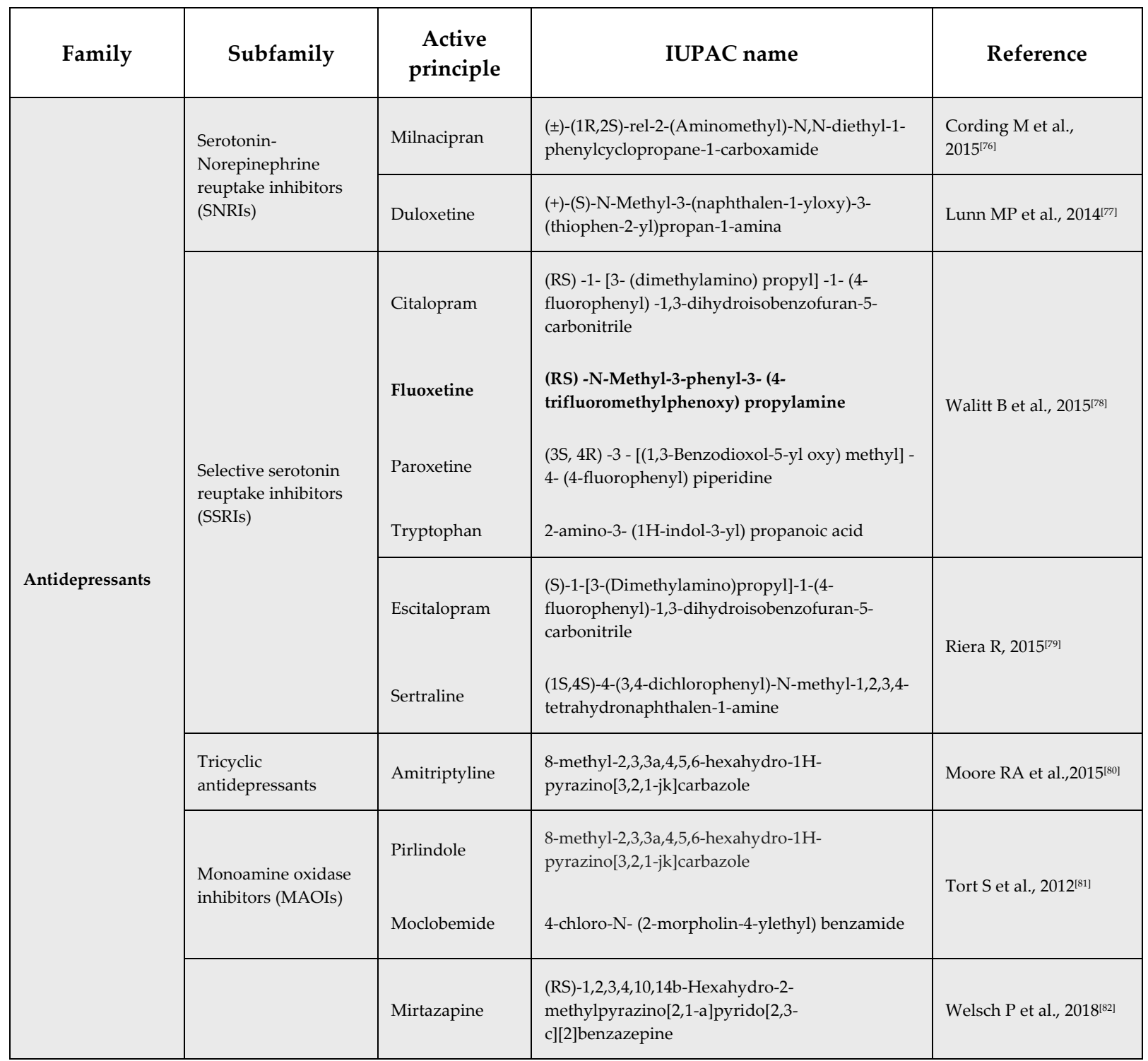




\begin{tabular}{|c|c|c|c|c|}
\hline \multirow{7}{*}{ Antiepileptics } & 1st Generation & Phenytoin & 5,5-diphenylimidazolidine-2,4-dione & Birse F et al., 2012 \\
\hline & \multirow{2}{*}{ 2nd Generation } & $\begin{array}{l}\text { Valproic acid } \\
\text { (Sodium } \\
\text { valproate) }\end{array}$ & 2-propylpentanoic acid & Gill D et al., 2011 ${ }^{[84]}$ \\
\hline & & Clonazepam & $\begin{array}{l}\text { 5-(2-Chlorophenyl)-7-nitro-1,3-dihydro-1,4- } \\
\text { benzodiazepin-2-one }\end{array}$ & $\begin{array}{l}\text { Corrigan R et al., } \\
2012^{[85]}\end{array}$ \\
\hline & \multirow{2}{*}{ 3rd Generation } & Pregabalin & (S)-3- (amynomethyl)-5-methylhexanoic acid & Derry S et al., 2016 ${ }^{[86]}$ \\
\hline & & Gabapentin & 2-[1-(amynomethyl)cyclohexyl]ethanoic acid & Wiffen PJ et al., 2017 \\
\hline & & Lacosamide & N2-acetyl-N-benzyl-D-homoserinamide & Hearn L et al., 2016 ${ }^{[88]}$ \\
\hline & & Topiramate & $\begin{array}{l}\text { 2,3: 4,5-Bis-O- (1-methylethylidene) -beta-D- } \\
\text { fructopyranose sulfamate }\end{array}$ & Wiffen PJ et al. , 2013 ${ }^{[89]}$ \\
\hline Antipsychotics & Atypical & Quetiapine & $\begin{array}{l}\text { 2- (2- (4-dibenzo }[\mathrm{b}, \mathrm{f}][1,4] \text { thiazepine-11-yl-1- } \\
\text { piperazinyl) ethoxy) ethanol }\end{array}$ & $\begin{array}{l}\text { Walitt B et al., } 2016 \\
\text { (Jun) }\end{array}$ \\
\hline Cannabinoids & Synthetic & Nabilone & $\begin{array}{l}\text { (6aR,10aR)-rel-1-Hydroxy-6,6-dimethyl-3-(2- } \\
\text { methyl-2-octanyl)-6,6a,7,8,10,10a-hexahydro-9H- } \\
\text { benzo[c]chromen-9-one }\end{array}$ & $\begin{array}{l}\text { Walitt B et al., } 2016 \\
(\mathrm{Jul})^{[91]}\end{array}$ \\
\hline \multirow{3}{*}{$\begin{array}{l}\text { Nonsteroidal anti- } \\
\text { inflammatory } \\
\text { drugs (NSAIDs) }\end{array}$} & $\begin{array}{l}\text { Selective inhibitor of } \\
\text { Cyclooxygenase } 2 \\
(\mathrm{COX}-2)\end{array}$ & Etoricoxib & $\begin{array}{l}\text { 5-cloro-6'-metil-3-[4-(metilsulfonil)fenil]-2,3'- } \\
\text { bipiridine }\end{array}$ & \multirow{3}{*}{ Derry S et al., 2017[92] } \\
\hline & $\begin{array}{l}\text { Inhibitor of } \\
\text { prostaglandin } \\
\text { synthesis }\end{array}$ & $\begin{array}{l}\text { Ibuprofen } \\
\text { Naproxen }\end{array}$ & $\begin{array}{l}\text { (RS)-2-(4-(2-Methylpropyl)phenyl)propanoic } \\
\text { acid } \\
\text { (+)-(S)-2-(6-Methoxynaphthalen-2-yl)propanoic } \\
\text { acid }\end{array}$ & \\
\hline & $\begin{array}{l}\text { Inhibitor of } \\
\text { Cicloxygenase } \\
\text { (COX-1 and COX-2) }\end{array}$ & Tenoxicam & $\begin{array}{l}\text { (3E)-3-[hydroxy(pyridin-2-ylamino)methylene] - } \\
\text { 2-methyl-2,3-dihydro-4H-thieno[2,3-e] } \\
\text { [1,2]thiazin-4-one 1,1-dioxide }\end{array}$ & \\
\hline Opioids & Semi synthetic & Oxycodone & $\begin{array}{l}(5 \mathrm{R}, 9 \mathrm{R}, 13 \mathrm{~S}, 14 \mathrm{~S}) \text {-4,5- } \alpha \text {-epoxy-14-hydroxy-3- } \\
\text { methoxy-17-methyl-morphinan-6-one }\end{array}$ & Gaskell H et al., 2016 ${ }^{[93]}$ \\
\hline
\end{tabular}

\subsubsection{Polypharmacy in ME/CFS}

Opposite to FM, no drug-based Cochrane review for the treatment of ME/CFS could be found. The three hits obtained by using the MeSH search terms "Chronic Fatigue Syndrome" were reviews on exercise, CBT (cognitive behaviour therapy) and Chineese herbs [94-96]. Therefore, we decided to use as reference papers to evaluate common drug-based ME/CFS therapies the recent reviews by Collatz et al., and Smith et al., [97,98]. Additional bibliography supporting the use of polypharmacy in ME/CFS was also included [48,97,99-107]. Similar to what has been described in the previous section 3.3.1. a documented summary of drugs commonly prescribed to ME/CFS patients that could impact miRNA screenings is shown in Table 4 together with active principles and IUPAC names. 
Table 4. Classification of drugs commonly prescribed to ME/CFS patients

\begin{tabular}{|c|c|c|c|c|}
\hline Family & Subfamily & Active principle & IUPAC name & Reference \\
\hline Anticonvulsants & 3rd Generation & $\begin{array}{l}\text { Gabapentin } \\
\text { Pregabalin }\end{array}$ & $\begin{array}{l}\text { 2-[1-(amynomethyl)cyclohexyl]ethanoic } \\
\text { acid } \\
\text { (S)-3- (amynomethyl)-5-methylhexanoic } \\
\text { acid }\end{array}$ & $\begin{array}{l}\text { Castro-Marrero J et al., } \\
2017^{[48]}\end{array}$ \\
\hline \multirow{5}{*}{ Antidepressants } & & Nafazodone & $\begin{array}{l}\text { 2-[3-[4-(3-chlorophenyl)piperazin-1- } \\
\text { yl]propyl]-5-ethyl-4-(2-phenoxyethyl)- } \\
\text { 1,2,4-triazol-3-one }\end{array}$ & Collatz A et al., 2016 \\
\hline & $\begin{array}{l}\text { Selective serotonin } \\
\text { reuptake inhibitors } \\
\text { (SSRIs) }\end{array}$ & $\begin{array}{l}\text { Bupropion } \\
\text { Citalopram } \\
\text { Escitalopram } \\
\text { Fluoxetine } \\
\text { Sertraline } \\
\text { Paroxetine }\end{array}$ & $\begin{array}{l}\text { (RS)-2-(tert-Butylamino)-1-(3- } \\
\text { chlorophenyl)propan-1-one } \\
\text { ((RS)-1-[3-(Dimethylamino)propyl]-1- } \\
\text { (4-fluorophenyl)-1,3- } \\
\text { dihydroisobenzofuran-5-carbonitrile } \\
\text { ((S)-1-[3-(Dimethylamino)propyl]-1-(4- } \\
\text { fluorophenyl)-1,3- } \\
\text { dihydroisobenzofuran-5-carbonitrile } \\
\text { (RS) -N-Methyl-3-phenyl-3- (4- } \\
\text { trifluoromethylphenoxy) propylamine } \\
\text { (1S,4S)-4-(3,4-dichlorophenyl)-N- } \\
\text { methyl-1,2,3,4-tetrahydronaphthalen-1- } \\
\text { amine } \\
\text { (3S, 4R) -3 - [(1,3-Benzodioxol-5-yl oxy) } \\
\text { methyl] -4- (4-fluorophenyl) piperidine }\end{array}$ & $\begin{array}{l}\text { Castro-Marrero J et al., } \\
2017^{[48]}\end{array}$ \\
\hline & \multirow{2}{*}{$\begin{array}{l}\text { Serotonin- } \\
\text { norepinephrine } \\
\text { reuptake inhibitors } \\
\text { (SNRIs) }\end{array}$} & Methylphenidate & Methyl phenyl(piperidin-2-yl)acetate & $\begin{array}{l}\text { Blockmans D and } \\
\text { Persoons P, 2016 } \\
\text { Castro-Marrero J et al., } \\
\text { 2017 }\end{array}$ \\
\hline & & $\begin{array}{l}\text { Duloxetine } \\
\text { Venlafaxine }\end{array}$ & $\begin{array}{l}\text { (+)-(S)-N-Methyl-3-(naphthalen-1- } \\
\text { yloxy)-3-(thiophen-2-yl)propan-1- } \\
\text { amine } \\
\text { (RS)-1-[2-dimethylamino-1-(4- } \\
\text { methoxyphenyl)-ethyl]cyclohexanol }\end{array}$ & $\begin{array}{l}\text { Castro-Marrero J et al., } \\
2017^{[48]}\end{array}$ \\
\hline & $\begin{array}{l}\text { Tricyclic } \\
\text { antidepressants }\end{array}$ & $\begin{array}{l}\text { Amitriptyline } \\
\text { Clomipramine } \\
\text { Desipramine } \\
\text { Doxepin } \\
\text { Imipramine } \\
\text { Nortriptyline }\end{array}$ & $\begin{array}{l}\text { 3- (10,11-dihydro-5H-dibenzo [a, d] } \\
\text { cycloheptene-5-ylidene) -N, N- } \\
\text { dimethyl-1-propanamine } \\
\text { 3-(2-chloro-5,6- } \\
\text { dihydrobenzo[b][1]benzazepin-11-yl)- } \\
\text { N,N-dimethylpropan-1-amine } \\
\text { 3-(5,6-dihydrobenzo[b][1]benzazepin- } \\
\text { 11-yl)-N-methylpropan-1-amine } \\
\text { (3E)-3-(6H-benzo[c][1]benzoxepin-11- } \\
\text { ylidene)-N,N-dimethylpropan-1-amine } \\
\text { 3-(5,6-dihydrobenzo[b][1]benzazepin- } \\
\text { 11-yl)-N,N-dimethylpropan-1-amine } \\
\text { 3-(5,6-dihydrodibenzo[2,1-b:2',1'- } \\
\text { f][7]annulen-11-ylidene)-N- } \\
\text { methylpropan-1-amine }\end{array}$ & $\begin{array}{l}\text { Castro-Marrero J et al., } \\
2017^{[48]}\end{array}$ \\
\hline
\end{tabular}




\begin{tabular}{|c|c|c|c|c|}
\hline & \multirow[t]{2}{*}{$\begin{array}{l}\text { Monoamine oxidase } \\
\text { inhibitors (MAOIs) }\end{array}$} & $\begin{array}{l}\text { Moclobemide } \\
\text { Phenelzine }\end{array}$ & $\begin{array}{l}\text { 4-chloro-N-(2-morpholin-4- } \\
\text { ylethyl)benzamide } \\
\text { 2-phenylethylhydrazine }\end{array}$ & $\begin{array}{l}\text { Collatz A et al., 2016 } \\
\text { Castro-Marrero J et al., } \\
\text { 2017/48] }\end{array}$ \\
\hline & & Selegiline & $\begin{array}{l}\text { (R)-N-methyl-N-(1-pheny lpropan-2- } \\
\text { yl)prop-1-yn-3-amine }\end{array}$ & $\begin{array}{l}\text { Castro-Marrero J et al., } \\
2017^{[48]}\end{array}$ \\
\hline & $\begin{array}{l}\text { Noradrenergic and } \\
\text { specific serotonin } \\
\text { antagonist (NaSSAs) }\end{array}$ & Mirtazapine & $\begin{array}{l}\text { (RS)-1,2,3,4,10,14b-Hexahydro-2- } \\
\text { methylpyrazino[2,1-a]pyrido[2,3- } \\
\text { c][2]benzazepine }\end{array}$ & $\begin{array}{l}\text { Castro-Marrero J et al., } \\
2017^{748]}\end{array}$ \\
\hline & $\begin{array}{l}\text { Monoaminergic } \\
\text { stabiliser }\end{array}$ & (-)-OSU-6162 & $\begin{array}{l}\text { (3S)-3-[3-(methylsulfonyl)phenyl]-1- } \\
\text { propylpiperidine }\end{array}$ & $\begin{array}{l}\text { Nilsson MKL et al., } \\
\text { 2017(100] }\end{array}$ \\
\hline \multirow[b]{2}{*}{ Antihypertensive } & $\begin{array}{l}\text { Stimulant to } \alpha 2- \\
\text { Receptors }\end{array}$ & $\begin{array}{l}\text { Clonidine } \\
\text { hydrochloride }\end{array}$ & $\begin{array}{l}\text { N-(2,6-dichlorophenyl)-4,5-dihydro- } \\
\text { 1H-imidazol-2-amine;hydrochloride }\end{array}$ & Collatz A et al., 2016 \\
\hline & $\begin{array}{l}\text { Angiotensin II receptor } \\
\text { agonist }\end{array}$ & $\begin{array}{l}\text { Olmesartan } \\
\text { medoxomil }\end{array}$ & $\begin{array}{l}\text { (5-metil-2-oxo-2H-1,3-dioxol-4-il)metil } \\
\text { 4-(2-hidroxipropan-2-il)-2-propil-1-(\{4- } \\
\text { [2-(2H-1,2,3,4-tetrazol-5- } \\
\text { il)fenil]fenil\}metil)-1H-imidazole-5- } \\
\text { carboxilato }\end{array}$ & Proal AD et al., 2013 $3^{[101]}$ \\
\hline \multirow[b]{2}{*}{ Antioxidant } & Fatty acid oxidant & L-Carnitine & $\begin{array}{l}\text { 3-Hydroxy-4- } \\
\text { (trimethylazaniumyl)butanoate }\end{array}$ & $\begin{array}{l}\text { Plioplys AV and Plioplys } \\
\text { S., 19971102] }\end{array}$ \\
\hline & $\begin{array}{l}\text { Ubiquinone } \\
\text { Re-Dox Agent }\end{array}$ & $\begin{array}{l}\text { CoQ10 } \\
\text { NADH }\end{array}$ & $\begin{array}{l}\text { [(2E,6E,10E,14E, 18E,22E,26E,30E,34E)- } \\
\text { 3,7,11,15,19,23,27,31,35,39- } \\
\text { Decamethyltetraconta- } \\
\text { 2,6,10,14,18,22,26,30,34,38-decaenyl]- } \\
\text { 5,6-dimethoxy-3-methylcyclohexa-2,5- } \\
\text { diene-1,4-dione } \\
\text { Nicotine adenine dinucleotide }\end{array}$ & $\begin{array}{l}\text { Castro-Marrero J et al., } \\
2015^{[103]}\end{array}$ \\
\hline \multirow[b]{2}{*}{ Antioxidant } & Omega-3 fatty acid & $\begin{array}{l}\alpha \text {-lipoic acid } \\
\text { Docosahexaenoic } \\
\text { acid(DHA) }\end{array}$ & $\begin{array}{l}\text { (R)-5-(1,2-dithiolan-3-yl)pentanoic acid } \\
\text { (4Z,7Z,10Z,13Z,16Z,19Z)-docosa- } \\
\text { 4,7,10,13,16,19-hexaenoic acid }\end{array}$ & \\
\hline & Vitamins & $\begin{array}{l}\text { Vitamin C } \\
\text { Folate (Vitamin B9) } \\
\text { Hydroxycobalamin } \\
\text { Vitamin B12) }\end{array}$ & $\begin{array}{l}\text { (2R)-2-[(1S)-1,2-dihydroxyethyl]-3,4- } \\
\text { dihydroxy-2H-furan-5-one } \\
\text { (2S)-2-[[4-[(2-Amino-4-oxo-1H-pteridin- } \\
6- \\
\text { yl)methylamino]benzoyl]amino]pentan } \\
\text { edioic acid } \\
\text { Co } \alpha \text {-[ } \alpha \text {-(5,6-dimethylbenzimidazolyl)]- } \\
\text { Co } \beta \text {-hydroxocobamide }\end{array}$ & $\begin{array}{l}\text { Castro-Marrero J et al., } \\
2017^{[48]}\end{array}$ \\
\hline \multirow{3}{*}{ Antiviral } & $\begin{array}{l}\text { Blocking adhesion and } \\
\text { viral penetration }\end{array}$ & Amantadine & 1-amino-adamantane & $\begin{array}{l}\text { Plioplys AV and Plioplys } \\
\text { S., } 1997^{102]}\end{array}$ \\
\hline & \multirow[b]{2}{*}{ Acid nucleics analogs } & Valganciclovir & $\begin{array}{l}\text { [2-[(2-amino-6-oxo-3H-purin-9- } \\
\text { yl)methoxy]-3-hydroxypropyl] (2S)-2- } \\
\text { amino-3-methylbutanoate }\end{array}$ & $\begin{array}{l}\text { Collatz A et al., 2016 } \\
\text { Castro-Marre } \\
\text { 2017"148] }\end{array}$ \\
\hline & & $\begin{array}{l}\text { Acyclovir } \\
\text { Valacyclovir }\end{array}$ & $\begin{array}{l}\text { 2-amino-9-(2-hydroxyethoxymethyl)- } \\
\text { 3H-purin-6-one } \\
\text { 2-[(2-amino-6-oxo-3H-purin-9- } \\
\text { yl)methoxy]ethyl (2S)-2-amino-3- } \\
\text { methylbutanoate }\end{array}$ & $\begin{array}{l}\text { Castro-Marrero J et al., } \\
2017^{748]}\end{array}$ \\
\hline
\end{tabular}




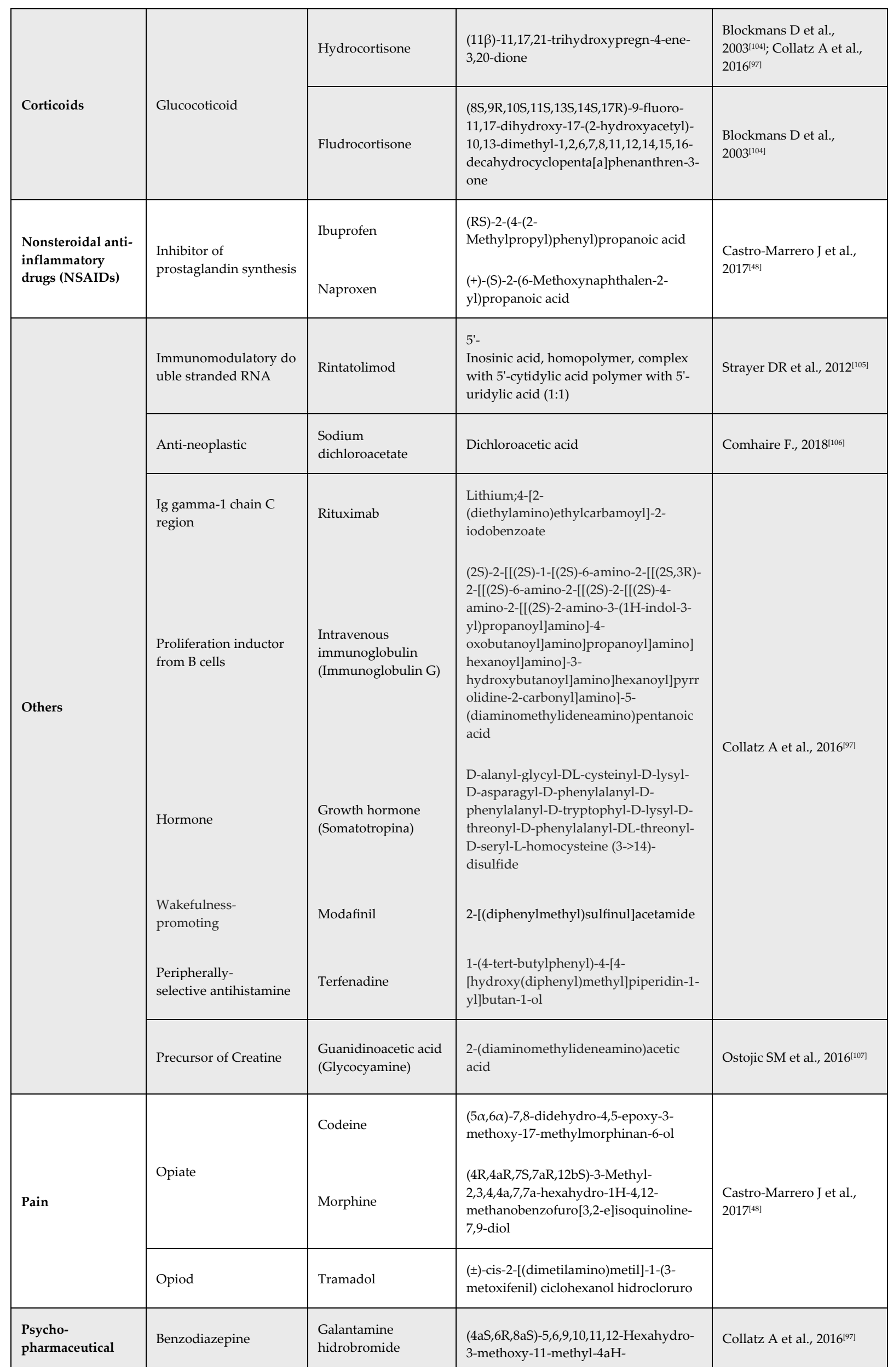




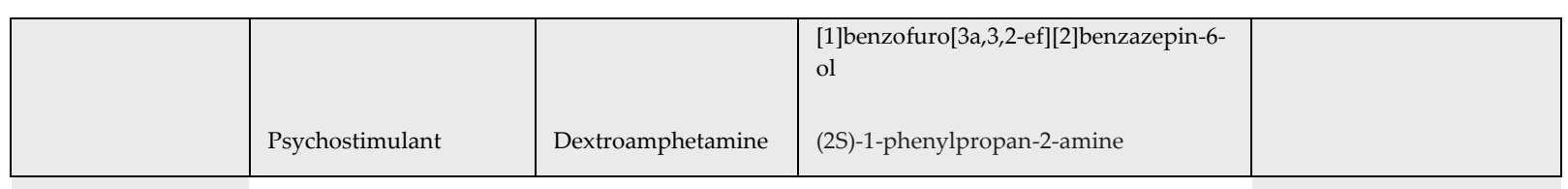

Although possibly not complete, Tables 3 and 4 include the most representative compounds to treat FM and ME/CFS according to the consulted authors [44,48,76-93,99-107]. Unexpectedly, a single IUPAC overlap, corresponding to the Selective Serotonin Reuptake Inhibitor (SSRI) Fluoxetine, was found for drugs commonly prescribed for FM and ME/CFS (in bold in Tables 3 \&4); indicating little prescription overlap at the IUPAC name level despite both groups of patients presenting common symptomatology. Special attention should be placed to common prescriptions as they may more readily allow identifying effects of drugs on miRNA levels over disease-related changes.

\section{3. miRNA-drug interactions in FM \& ME/CFS}

With the intention to discriminate whether the miRNomes proposed to associate with FM, or with ME/CFS, are derived from drug intake differences between the patient and control groups, we performed a screen of drugs that could alter any of the miRNAs in these miRNomes using the SM2miR web server [65] and each of the individual DE miRNAs or disease miRNome as the input, as previously detailed in the Methods section.

The SM2miR drug output file (Table S2) was contrasted with the FM \& ME/CFS polypharmacy tables (Tables $3 \& 4$ ), finding that five of the commonly prescribed drugs for FM or ME/CFS (DHA, fluoxetine, glucocorticoids, morphine and valproate) are estimated to alter the levels of one or more of the miRNAs found DE in FM or ME/CFS screenings (potential disease-associated miRNomes) and therefore these drugs could constitute confounding variables of the assay (Table 5) [108-113]. Overlapping tendencies may suggest that the detected differences between studied groups associate with treatment rather than constituting potential biomarkers of disease, while opposed tendencies might reflect additional factors leading to differential expression other than drug intake, disease status included. Importantly, as summarized in Table 5, the expression of miRNA-27b reported in miRNomes of both FM and ME/CFS in more than one report $[67,74]$ is affected by the only overlapping compound commonly prescribed for treatment of both diseases (Fluoxetine), indicating a potential druginterference effect. Three additional miRNAs reported as miRNomes of ME/CFS by more than one study (miR-26a, miR-126 \& miR-191) are also affected by drugs frequently prescribed to ME/CFS patients and therefore special attention should be paid when interpreting miRNome results including these miRNAs.

It must be pointed out that, in an effort to complete the search as much as possible the list of DE miRNAs in FM and ME/CFS used in the SM2miR search not only included the miRNAs listed in Tables $1 \& 2$ but also those documented in supplementary tables of the listed literature [67-74]. 
Table 5. Effect of FM \& ME/CFS polypharmacy on miRNomes associated to disease

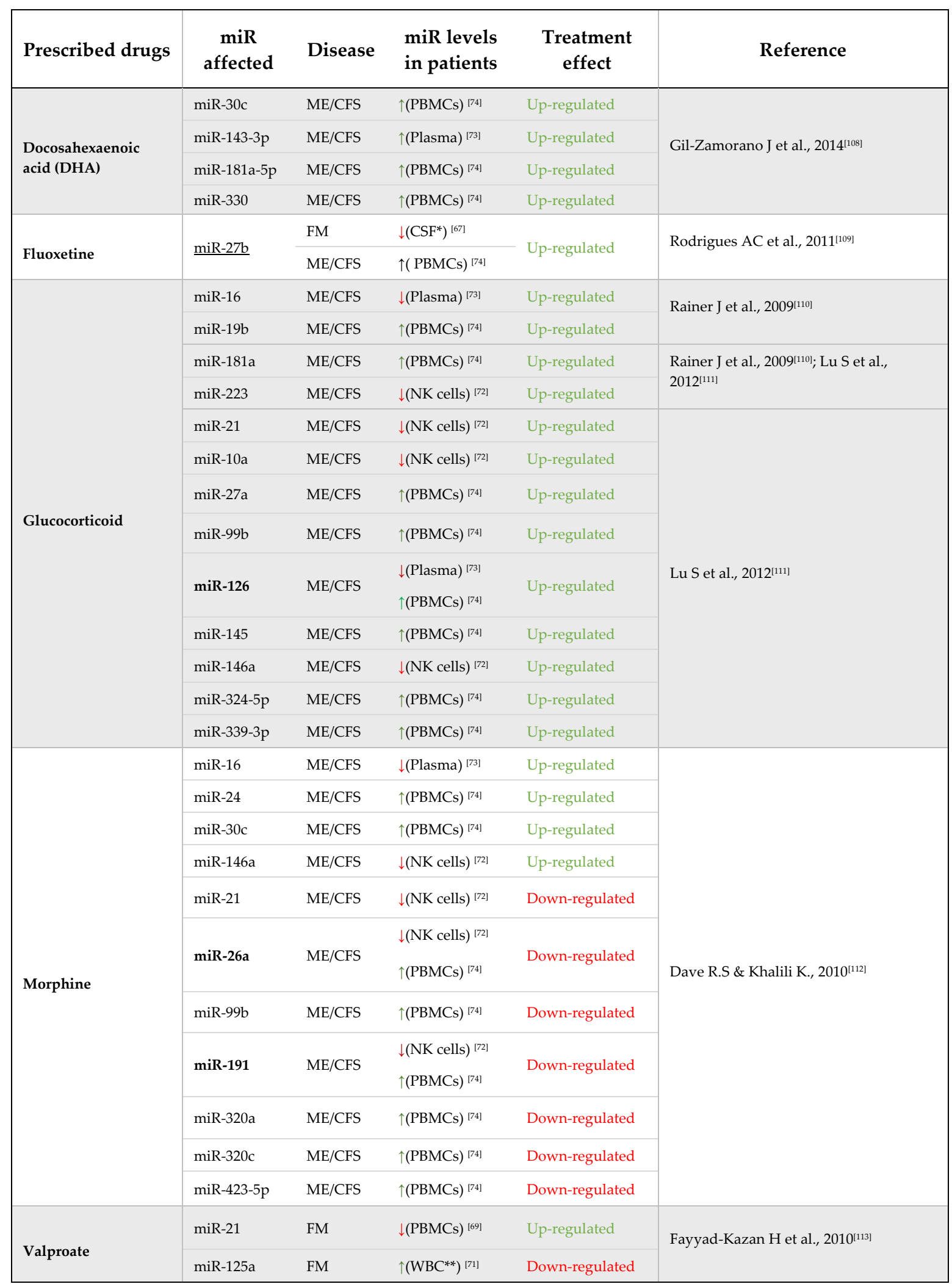




\subsection{Drug-disease interactions based on FM and ME/CFS miRNomes}

To evaluate potential biased responses of FM and ME/CFS patients to pharmacotherapy in general, due to their DE miRNA profiles, we searched for diseases commonly treated with smallmolecule drugs that depend on gene sets linked to FM or ME/CFS miRNomes (miRNA-gene-drug datasets). With this purpose individual DE miRNAs in FM or ME/CFS were used as input in the Pharmaco-miR web search tool [50]. The output constituted a list of genes whose expression is dependent on FM and ME/CFS DE miRNAs (Table S3) and a third column facilitating small molecule drug associations for these gene lists. Among the 709 small molecules linked to FM miRNome only 595 appeared registered in Drugbank database. And, out of the 668 small molecules associating with ME/CFS miRNomes, 557 appeared registered in Drugbank [64].

Finally, Drugbank numbers of these small molecules were used as the input to search repoDB, a database of small drugs developed by Brown \& Patel to facilitate screenings for drug repositioning [66]. The results (Table S3, miRNome-drug-disease tabs) show 1480 and 1455 diseases treated with small molecules respectively associating with FM or ME/CFS miRNomes after filtering out duplications. Out of these diseases potentially impacting individualized medicine programs for FM and ME/CFS patients, more than 30\% corresponded to cancer of some type. Within cancer, $13 \%$ corresponded to lymphoma, and $14 \%$ to lymphoma plus leukaemia. This seems to indicate that quite possibly FM and ME/CFS patients may respond differently to treatments for these diseases with respect to non-FM, non-ME/CFS patients and, therefore, attention is advised in individualized medicine programs for the treatment of these cancers in the case of FM and CFS/ME patients.

\section{Discussion}

This paper evaluates for the first time the relationship between commonly prescribed drugs for FM and ME/CFS and miRNA expression and compares these profiles to FM and ME/CFS reported miRNomes in an effort to discern miRNAs presenting differential expression due to medication from differences more likely related to disease. The resources used in this study are limited and therefore it is expected that the evidence presented here will be refined as more data becomes available. The topic is not exclusive of FM and ME/CFS as it can be extended to any other study evaluating miRNomes associated to disease. However, the fact that FM and ME/CFS patients are usually polymedicated to palliate the multiple symptoms that associate with these illnesses, extends this concern to higher levels, particularly demanding careful registry of study participants medication, when restrictive medication inclusion criteria is not an option. In this sense, the ME/CFS Common Data Elements initiative [49] has made publicly available medication guidelines and CRFs at the disposition of researchers which may help standardize medication registry in ME/CFS studies.

Although some researchers have expressed their concern of the impact of drug use by FM and $\mathrm{ME} / \mathrm{CFS}$ patients on the study of molecular markers and although recent work in the area is already reporting the medication used by participants [71,113], the information of registered drugs is not yet used to evaluate potential interference or bias of results. To elevate biomarker screenings of FM and ME/CFS based on miRNA profiles, complex stratified analysis to filter out potential drug and other confounding variables will be required. The complexity and limitations of this analysis is served by the fact that miRNA expression respond to many cues, such as exercise and diet, hormone, sex and aging [38-43].

A commonly used approach, to minimize confounding variables, although not free of certain difficulties for sampling, is to set restrictive inclusion criteria including sex selection, narrow age range, and BMI. This is important in miRNA screenings as these parameters are known to affect miRNA profiles [114]. Additional sampling details such as fasting blood draw and selection of sedentary healthy controls may improve study outcomes. Some authors have even taken into account time of blood collection to reduce circadian variation [71], however, it may not be possible to eliminate polypharmacy, particularly in studies including severely affected FM and ME/CFS patients.

Prescriptions for other common health problems such as diabetes or high cholesterol, diet supplements and some recreational drugs alter the expression of some miRNAs in FM \& ME/CFS miRNomes (Table S4) [109,116-127]. Hormones and other natural compounds also impact FM \& 
ME/CFS miRNomes (Table S5)[108,128-138], stressing the necessity for researchers to collect complete medical histories of participants to accurately evaluate miRNAs as biomarkers of these diseases.

On another end, despite FM \& ME/CFS miRNomes relate to disease or derive from chronic polypharmacy use, DE miRs should represent a relevant factor to take into account when treatments for other diseases such as cancer are due. Here, we performed an analysis of the diseases whose treatment response could differ in the context of FM \& ME/CFS miRNomes, finding a broad range of them. The major representation of cancer (above 35\%) might merely reflect the fact that more studies are registered in the field, biasing databases. Importantly, a relevant number of hits associated with lymphoma, a type of cancer appearing at higher incidence among ME/CFS patients [139], possibly due to associated immune dysfunctions of this disease.

Personalized medicine programs considering miRNome backgrounds may more adequately select effective treatments with reduced side effects. It is therefore envisioned that future improved therapeutic analysis, including pharmacogenomics and pharmacoepigenomics (precision medicine programs), will rely on complex software tools fed with large datasets. Further miRNA profiling studies including larger number of samples are required to build on the scarce available FM \& ME/CFS miRNome data. Since technical variability in miRNA qPCR replicates has been documented, with TaqMan overweighing qScript PCR [140], future studies should include repeated independent measures or either use alternative enzyme-free approaches such as NanoString [141].

In general we have evaluated the effects of polypharmacy and miRNomes at individual levels, meaning that the information obtained here corresponds to the effects of a single drug on DE miRNAs or the impact of an individual miRNA on drug performance, however, the effects of combined therapies on miRNA profiles or sets of DE miRNAs on drug-response may not replicate or be additive of single events, highlighting the limitation of our study. In addition, most molecular data come from analysis of blood or other body fluid samples and more sparely from non-cancerous solid tissues, limiting the validity of results, as miRNA profiles are known to be tissue restricted [142]. Drug-assays are performed in either animal models or tumor cell lines leading to results that may not replicate in other systems; many miRNAs are primate or human specific $[63,143]$.

In summary, as larger data sets become available to nurture databanks, biomarker discovery will be facilitated and personalized medicine programs will be refined, upgrading current diagnostic tools and clinical treatments. Drug-transcriptome interactions are key factors in either context, particularly in diseases subject to polypharmacy such as FM and ME/CFS.

\section{Conclusions}

The analysis presented here strongly supports a potential impact of FM and ME/CFS polypharmacy in the discovery of miRNomes associating with these diseases. Additional carefully designed studies including larger cohorts of patients providing complete drug registry to permit stratified analysis will facilitate identification of DE miRNAs linked to these diseases.

FM and ME/CFS miRNomes may predispose patients to respond differently to a large variety of drug-based treatments, including those used for a large number of cancers, highlighting the importance of considering this epigenomic bias in refined personalized programs towards improving patient's response to clinical treatments while minimizing toxicity. It is estimated that future more sophisticated informatic tools will help with these predictions, however, the paucity of molecular studies in FM and ME/CFS currently limits their development.

Supplementary Materials: The following are available online at www.mdpi.com/xxx/s1, Table S1: PRISMA based search of FM and ME/CFS miRNA profiling studies, Table S2: FM and ME/CFS miRNomes SM2miR-based drug search, Table S3: FM and ME/CFS miRNomes PharmacomiR-based gene-drug associations and repoDBdrug-disease screening, Table S4: Effect of additional drugs, supplements and recreational drugs on FM \& ME/CFS miRNomes and Table S5: Effect of hormones and other natural compounds on FM \& ME/CFS miRNomes.

Abreviations: Fibromyalgia (FM); Myalgic Encephalomyelitis/Chronic Fatigue Syndrome (ME/CFS); microRNAs (miRs); Differentially Expressed (DE); American College of Rheumatology (ACR); Gene Ontology 
(GO);Preferred Reporting Items for Systematic Reviews and Meta-Analyses criteria (PRISMA); International Classification of Diseases (ICD); NonSteroidal Anti-Inflammatory Drugs (NSAIDs); Cognitive Behaviour Therapy (CBT); International Union of Pure and Applied Chemistry (IUPAC); Concept Unique Identifiers (CUI); National Clinical Trial (NCT); Common Data Elements (CDE); Case Report Forms (CRFs); National Institute of Health (NIH); Absorption, Distribution, Metabolism, and Excretion (ADME); Selective Serotonin Reuptake Inhibitor (SSRI); Serotonin-Norepinephrine reuptake inhibitors (SNRIs); Monoamine oxidase inhibitors (MAOIs).

Author Contributions: Individual author contributions are as follows: conceptualization, E.O.; methodology, E.A-P.; T. S-F.; T.O.; L.N.\& E.O; software, formal analysis \& data curation, E.A-P.; T. S-F.; T.O.; L.N.\& E.O.; writing - original draft preparation, E.O.; writing - review and editing, E.A-P.; T. S-F.; T.O.; L.N.\& E.O.; funding acquisition, E.O.

Funding: This research was funded by Universidad Católica de Valencia San Vicente Mártir research grant 2018270-01 to E.O.

Conflicts of Interest: The authors declare no conflict of interest. The funders had no role in the design of the study; in the collection, analyses, or interpretation of data; in the writing of the manuscript, or in the decision to publish the results.

\section{References}

1. Wolfe F, Smythe HA, Yunus MB, Bennett RM, Bombardier C, Goldenberg DL et al. The American College of Rheumatology 1990 Criteria for the Classification of fibromyalgia. Report of the Multicenter Criteria Committee. Arthritis Rheum. 1990;33(2):160-72.doi: 10.1002/art.1780330203.

2. Wolfe F, Clauw DJ, Fitzcharles MA, et al. The American College of Rheumatology preliminary diagnostic criteria for fibromyalgia and measurement of symptom severity. Arthritis Care Res (Hoboken) 2010; 62(5): 600-610.doi: 10.1002/acr.20140.

3. Jahan F, Nanji K, Qidwai W, Qasim R. Fibromyalgia syndrome: an overview of pathophysiology, diagnosis and management. Oman Med J. 2012;27(3):192-5.doi: 10.5001/omj.2012.44.

4. Arnold LM, Bennett RM, Crofford LJ, Dean LE, Clauw DJ, Goldenberg DL, et al. AAPT Diagnostic Criteria for Fibromyalgia. J Pain. 2018 Nov 16. pii:S1526-5900(18)30832-0. doi: 10.1016/j.jpain.2018.10.008.

5. Abbi B, Natelson $\mathrm{BH}$. Is chronic fatigue syndrome the same illness as fibromyalgia: evaluating the 'single syndrome' hypothesis. QJM. 2013;106(1):3-9.doi: 10.1093/qjmed/hcs156.

6. Fukuda K, Straus SE, Hickie I, Sharpe MC, Dobbins JG, Komaroff A. The chronic fatigue syndrome: a comprehensive approach to its definition and study. International Chronic Fatigue Syndrome Study Group. Ann Intern Med. 1994 Dec 15;121(12):953-9.

7. Carruthers BM, Jain, AK, De Meirleir KL, Peterson DL, Klimas NG, Lerner A, et al. Myalgic Encephalomyelitis/Chronic Fatigue Syndrome: Clinical Working Case Definition, Diagnostic and Treatment Protocols. Journal of Chronic Fatigue Syndrome 2003;11 (2): 7-115. doi:10.1300/J092v11n01_02

8. Carruthers BM, van de Sande MI, De Meirleir KL, Klimas NG, Broderick G, Mitchell T, et al. Myalgic encephalomyelitis: International Consensus Criteria. J Intern Med. 2011;270(4):327-38. doi: 10.1111/j.13652796.2011.02428.x.

9. Committee on the Diagnostic Criteria for Myalgic Encephalomyelitis/Chronic Fatigue Syndrome, Board on the Health of Select Populations, Institute of Medicine. Beyond Myalgic Encephalomyelitis/Chronic Fatigue Syndrome: Redefining an Illness. Washington (DC): National Academies Press (US); 2015 Feb 10.PubMed PMID: 25695122.

10. Clayton EW. Beyond myalgic encephalomyelitis/chronic fatigue syndrome: a IOM report on redefining an illness. JAMA. 2015 Mar 17;313(11):1101-2. doi:10.1001/jama.2015.1346. PubMed PMID: 25668027.

11. Jason LA, McManimen S, Sunnquist M, Newton JL, Strand EB. Clinical Criteria Versus a Possible Research Case Definition in Chronic Fatigue Syndrome/Myalgic Encephalomyelitis. Fatigue. 2017;5(2):89-102. doi: 10.1080/21641846.2017.1299077.

12. Boerma T, Harrison J, Jakob R, Mathers C, Schmider A, Weber S. Revising the ICD: explaining the WHO approach. Lancet. 2016;388(10059):2476-2477.doi: 10.1016/S0140-6736(16)31851-7.

13. Jones GT, Atzeni F, Beasley M, Flüß E, Sarzi-Puttini P, Macfarlane GJ. The prevalence of fibromyalgia in the general population - a comparison of the American College of Rheumatology 1990, 2010 and modified 2010 classification criteria. Arthritis Rheumatol. 2014 Oct 16. doi: 10.1002/art.38905. 
14. Cabo-Meseguer A, Cerdá-Olmedo G, Trillo-Mata JL. Fibromyalgia: Prevalence, epidemiologic profiles and economic costs. Med Clin (Barc). 2017 Nov 22;149(10):441-448. doi: 10.1016/j.medcli.2017.06.008.

15. Reyes M, Nisenbaum R, Hoaglin DC, Unger ER, Emmons C, Randall B, et al. Prevalence and incidence of chronic fatigue syndrome in Wichita, Kansas. Arch Intern Med. 2003;163(13):1530-6.

16. Jason LA, Richman JA, Rademaker AW, Jordan KM, Plioplys AV, Taylor RR, et al. A community-based study of chronic fatigue syndrome. Arch Intern Med. 1999;159(18):2129-37.

17. Estévez-López F, Castro-Marrero J, Wang X, Bakken IJ, Ivanovs A, Nacul L, et al; European Network on ME/CFS (EUROMENE). Prevalence and incidence of myalgic encephalomyelitis/chronic fatigue syndrome in Europe-the Euro-epiME study from the European network EUROMENE: a protocol for a systematic review. BMJ Open. 2018 Sep 4;8(9):e020817. doi: 10.1136/bmjopen-2017-020817.

18. Crawley E. Pediatric chronic fatigue syndrome: current perspectives. Pediatric Health Med Ther. 2018 Mar 29;9:27-33. doi: 10.2147/PHMT.S126253.

19. McManimen SL, Jason LA. Post-Exertional Malaise in Patients with ME and CFS with Comorbid Fibromyalgia. SRL Neurol Neurosurg. 2017; 3(1):22-27.

20. Naschitz JE, Rozenbaum M, Rosner I, Sabo E, Priselac RM, Shaviv N, et al. Cardiovascular response to upright tilt in fibromyalgia differs from that in chronic fatigue syndrome. J Rheumatol 2001; 28:1356-60.

21. Naschitz JE, Slobodin G, Sharif D, Fields M, Isseroff H, Sabo E, et al. Electrocardiographic QT interval and cardiovascular reactivity in fibromyalgia differ from chronic fatigue syndrome. Eur J Intern Med 2008; 19:187-91. doi: 10.1016/j.ejim.2007.08.003.

22. Korszun A, Sackett-Lundeen L, Papadopoulos E, Brucksch C, Masterson L, Engelberg NC, et al. Melatonin levels in women with fibromyalgia and chronic fatigue syndrome. J Rheumatol 1999; 26:2675-80. 21.

23. Crofford LJ, Young EA, Engleberg NC, Korszun A, Brucksch CB, McClure LA, et al. Basal circadian and pulsatile ACTH and cortisol secretion in patients with fibromyalgia and/or chronic fatigue syndrome. Brain Behav Immun 2004;18:314-25.doi: 10.1016/j.bbi.2003.12.011.

24. Light AR, Bateman L, Jo D, Hughen RW, Vanhaitsma TA, White AT, Light KC. Gene expression alterations at baseline and following moderate exercise in patients with Chronic Fatigue Syndrome and Fibromyalgia Syndrome. J Intern Med. 2012;271(1):64-81.doi: 10.1111/j.1365-2796.2011.02405.x.

25. Scheibenbogen C, Freitag H, Blanco J, Capelli E, Lacerda E, Authier J, et al. The European ME/CFS Biomarker Landscape project: an initiative of the European network EUROMENE. J Transl Med. 2017 Jul 26;15(1):162. doi: 10.1186/s12967-017-1263-z.

26. Xiao C, Rajewsky K. MicroRNA control in the immune system: basic principles. Cell. 2009; 136(1):26--36. doi: 10.1016/j.cell.2008.12.027 Erratum in: Cell. 2009; 137(2):380. PMID: 19135886

27. Emde A, Hornstein E. miRNAs at the interface of cellular stress and disease. EMBO J. 2014; 33-(13):142837. doi: 10.15252/embj.201488142 PMID: 24867813

28. Puik JR, Meijer LL, Le Large TY, Prado MM, Frampton AE, Kazemier G, et al. miRNA profiling for diagnosis, prognosis and stratification of cancer treatment in cholangiocarcinoma. Pharmacogenomics. 2017 Sep;18(14):1343-1358. doi: 10.2217/pgs-2017-0010.

29. Filipowicz W, Bhattacharyya SN, Sonenberg N. Mechanisms of post-transcriptional regulation by microRNAs: are the answers in sight? Nat Rev Genet. 2008; 9(2):102-14. doi: 10.1038/nrg2290.

30. Vasudevan S. Posttranscriptional upregulation by microRNAs. Wiley Interdiscip Rev RNA. 2012( 3):31130.doi: 10.1002/wrna.121 PMID: 22072587.

31. Xia T, Li J, Cheng H, Zhang C, Zhang Y. Small-Molecule Regulators of MicroRNAs in Biomedicine. Drug Dev Res. 2015 Nov;76(7):375-81. doi: 10.1002/ddr.21271.

32. Cha W, Fan R, Miao Y, Zhou Y, Qin C, Shan X, Wan X, Cui T. MicroRNAs as novel endogenous targets for regulation and therapeutic treatments. Medchemcomm. 2017 Dec 11;9(3):396-408. doi: 10.1039/c7md00285h.

33. Melo S, Villanueva A, Moutinho C, Davalos V, Spizzo R, Ivan C, et al. Small molecule enoxacin is a cancerspecific growth inhibitor that acts by enhancing TAR RNA-binding protein 2-mediated microRNA processing. Proc Natl Acad Sci U S A. 2011 Mar 15;108(11):4394-9. doi: 10.1073/pnas.1014720108. Epub 2011 Feb 28. Erratum in: Proc Natl Acad Sci U S A. 2017 Jan 17.

34. Watashi K, Yeung ML, Starost MF, Hosmane RS, Jeang KT. Identification of small molecules that suppress microRNA function and reverse tumorigenesis. J Biol Chem. 2010 Aug 6;285(32):24707-16. doi: 10.1074/jbc.M109.062976.

35. Qiu C, Chen G, Cui Q. Towards the understanding of microRNA and environmental factor interactions and their relationships to human diseases. Sci Rep. 2012;2:318. doi: 10.1038/srep00318. 
36. Yang Q, Qiu C, Yang J, Wu Q, Cui Q. miREnvironment database: providing a bridge for microRNAs, environmental factors and phenotypes. Bioinformatics. 2011 Dec 1;27(23):3329-30. doi: 10.1093/bioinformatics/btr556.

37. Luo H, Lan W, Chen Q, Wang Z, Liu Z, Yue X, Zhu L. Inferring microRNA-Environmental Factor Interactions Based on Multiple Biological Information Fusion. Molecules. 2018 Sep 24;23(10). pii: E2439. doi:10.3390/molecules23102439.

38. Peng C, Wang YL. Editorial: MicroRNAs as New Players in Endocrinology. Front Endocrinol (Lausanne). 2018 Aug 17;9:459. doi: 10.3389/fendo.2018.00459.

39. Smith-Vikos T, Slack FJ. MicroRNAs and their roles in aging. J Cell Sci. 2012 Jan 1;125(Pt 1):7-17. doi: 10.1242/jcs.099200.

40. Rani A, O'Shea A, Ianov L, Cohen RA, Woods AJ, Foster TC. miRNA in Circulating Microvesicles as Biomarkers for Age-Related Cognitive Decline. Front Aging Neurosci. 2017 Oct 4;9:323. doi: 10.3389/fnagi.2017.00323.

41. Vienberg S, Geiger J, Madsen S, Dalgaard LT. MicroRNAs in metabolism. Acta Physiol (Oxf). 2017 Feb;219(2):346-361. doi: 10.1111/apha.12681.

42. Lopez S, Bermudez B, Montserrat-de la Paz S, Abia R, Muriana FJG. A microRNA expression signature of the postprandial state in response to a high-saturated-fat challenge. J Nutr Biochem. 2018 Jul;57:45-55. doi:10.1016/j.jnutbio.2018.03.010.

43. Sapp RM, Shill DD, Roth SM, Hagberg JM. Circulating microRNAs in acute and chronic exercise: more than mere biomarkers. J Appl Physiol (1985). 2017 Mar 1;122(3):702-717. doi: 10.1152/japplphysiol.00982.2016.

44. Häuser W, Welsch P, Klose P, Derry S, Straube S, Wiffen PJ, et al. Pharmacological therapies for fibromyalgia in adults - an overview of Cochrane Reviews. Cochrane Database of Systematic Reviews 2018, Issue 10. Art. No.: CD013151. DOI: 10.1002/14651858.CD013151.

45. Thorpe J, ShumB, Moore RA, Wiffen PJ, Gilron I. Combination pharmacotherapy for the treatment of fibromyalgia in adults. Cochrane Database of Systematic Reviews 2018, Issue 2. Art. No.: CD010585. DOI: 10.1002/14651858.CD010585.pub2.

46. Collatz A, Johnston SC, Staines DR, Marshall-Gradisnik SM. A Systematic Review of Drug Therapies for Chronic Fatigue Syndrome/Myalgic Encephalomyelitis. Clin Ther. 2016 Jun;38(6):1263-1271.e9. doi: 10.1016/j.clinthera.2016.04.038.

47. Smith ME, Haney E, McDonagh M, Pappas M, Daeges M, Wasson N, et al. Treatment of Myalgic Encephalomyelitis/Chronic Fatigue Syndrome: A Systematic Review for a National Institutes of Health Pathways to Prevention Workshop. Ann Intern Med. 2015 Jun 16;162(12):841-50. doi: 10.7326/M15-0114.

48. Castro-Marrero J, Sáez-Francàs N, Santillo D, Alegre J. Treatment and management of chronic fatigue syndrome/myalgic encephalomyelitis: all roads lead to Rome. Br J Pharmacol. 2017 Mar;174(5):345-369. doi: 10.1111/bph.13702.

49. NINDS. Common Data Available on line: https://www.commondataelements.ninds.nih.gov/MECFS.aspx\#tab=Data_Standards (accessed on 15 November 2018).

50. Rukov JL, Wilentzik R, Jaffe I, Vinther J, Shomron N. Pharmaco-miR: linking microRNAs and drug effects. Brief Bioinform. 2014 Jul;15(4):648-59. doi:10.1093/bib/bbs082. Epub 2013 Jan 31.

51. Russo F, Di Bella S, Bonnici V, Laganà A, Rainaldi G, Pellegrini M, et al. A knowledge base for the discovery of function, diagnostic potential and drug effects on cellular and extracellular miRNAs. BMC Genomics.2014;15 Suppl 3:S4. doi: 10.1186/1471-2164-15-S3-S4.

52. Zhang G, Nebert DW. Personalized medicine: Genetic risk prediction of drug response. Pharmacol Ther. 2017 Jul;175:75-90. doi:10.1016/j.pharmthera.2017.02.036.

53. Lloyd RA, Hotham E, Hall C, Williams M, Suppiah V. Pharmacogenomics and Patient Treatment Parameters to Opioid Treatment in Chronic Pain: A Focus on Morphine, Oxycodone, Tramadol, and Fentanyl. Pain Med. 2017 Dec 1;18(12):2369-2387. doi: 10.1093/pm/pnw317.Shomron N. MicroRNAs and pharmacogenomics. Pharmacogenomics. 2010 May;11(5):629-32. doi: 10.2217/pgs.10.26.

54. Rukov JL, Shomron N. MicroRNA pharmacogenomics: post-transcriptional regulation of drug response. Trends Mol Med. 2011 Aug;17(8):412-23. doi:10.1016/j.molmed.2011.04.003.

55. Bracco L, Kearsey J. The relevance of alternative RNA splicing to pharmacogenomics. Trends Biotechnol. 2003 Aug;21(8):346-53. 
56. Ivanov M, Kacevska M, Ingelman-Sundberg M. Epigenomics and interindividual differences in drug response. Clin Pharmacol Ther. 2012 Dec;92(6):727-36. doi:10.1038/clpt.2012.152.

57. Hu H, Baines C. Recent insights into 3 underrecognized conditions: Myalgic encephalomyelitis-chronic fatigue syndrome, fibromyalgia, and environmental sensitivities-multiple chemical sensitivity. Can Fam Physician. 2018 Jun;64(6):413-415.

58. Liberati A, Altman DG, Tetzlaff J, Mulrow C, Gøtzsche PC, Ioannidis JP, et al. The PRISMA statement for reporting systematic reviews and meta-analyses of studies that evaluate health care interventions: explanation and elaboration. PLoS Med. 2009 Jul 21;6(7):e1000100. doi: 10.1371/journal.pmed.1000100.

59. PubMed US National Library of Medicine National Institutes of Health. Available on line: https://www.ncbi.nlm.nih.gov/pubmed (accessed on 10 October 2018).

60. FECYT. Fundación Española para la Ciencia y la Tecnología. Web of Science (WOS). Available on line: https://www.fecyt.es/es/recurso/web-science (accessed on 10 October 2018).

61. Pubmed Labs. Bethesda (MD): National Library of Medicine (US), National Center for Biotechnology Information; 2018 - [cited 201812 01]. Available from: https://www.ncbi.nlm.nih.gov/labs/pubmed/ (accessed on 10 October 2018).

62. Cochrane Library. Available on line: https://www.cochranelibrary.com (accessed on 11 October 2018).

63. Kozomara A, Griffiths-Jones S. miRBase: integrating microRNA annotation and deep-sequencing data. Nucleic Acids Res. 2011 Jan;39(Database issue):D152-7. doi: 10.1093/nar/gkq1027.

64. Wishart DS, Feunang YD, Guo AC, Lo EJ, Marcu A, Grant JR, et al. DrugBank 5.0: a major update to the DrugBank database for 2018. Nucleic Acids Res. 2018 Jan 4;46(D1):D1074-D1082. doi: 10.1093/nar/gkx1037.

65. Liu X, Wang S, Meng F, Wang J, Zhang Y, Dai E, Yu X, Li X, Jiang W. SM2miR: a database of the experimentally validated small molecules' effects on microRNA expression. Bioinformatics. 2013 Feb 1;29(3):409-11. doi:10.1093/bioinformatics/bts698.

66. Brown AS, Patel CJ. A standard database for drug repositioning. Sci Data. 2017 Mar 14;4:170029. doi: 10.1038/sdata.2017.29.

67. Bjersing JL, Lundborg C, Bokarewa MI, Mannerkorpi K. Profile of cerebrospinal microRNAs in fibromyalgia. PLoS One. 2013 Oct 25;8(10):e78762. doi:10.1371/journal.pone.0078762.

68. Bjersing JL, Bokarewa MI, Mannerkorpi K. Profile of circulating microRNAs in fibromyalgia and their relation to symptom severity: an exploratory study. Rheumatol Int. 2015 Apr;35(4):635-42. doi: 10.1007/s00296-014-3139-3.

69. Cerdá-Olmedo G, Mena-Durán AV, Monsalve V, Oltra E. Identification of a microRNA signature for the diagnosis of fibromyalgia. PLoS One. 2015 Mar 24;10(3):e0121903. doi: 10.1371/journal.pone.0121903.

70. Masotti A, Baldassarre A, Guzzo MP, Iannuccelli C, Barbato C, Di Franco M. Circulating microRNA Profiles as Liquid Biopsies for the Characterization and Diagnosis of Fibromyalgia Syndrome. Mol Neurobiol. 2017 Nov;54(9):7129-7136. doi:10.1007/s12035-016-0235-2.

71. Leinders M, Doppler K, Klein T, Deckart M, Rittner H, Sommer C, Üçeyler N. Increased cutaneous miRlet-7d expression correlates with small nerve fiber pathology in patients with fibromyalgia syndrome. Pain. 2016 Nov;157(11):2493-2503.

72. Brenu EW, Ashton KJ, van Driel M, Staines DR, Peterson D, Atkinson GM, et al. Cytotoxic lymphocyte microRNAs as prospective biomarkers for Chronic Fatigue Syndrome/Myalgic Encephalomyelitis. J Affect Disord. 2012 Dec 10;141(2-3):261-9. doi: 10.1016/j.jad.2012.03.037.

73. Brenu EW, Ashton KJ, Batovska J, Staines DR, Marshall-Gradisnik SM. High-throughput sequencing of plasma microRNA in chronic fatigue syndrome/myalgic encephalomyelitis. PLoS One. 2014 Sep 19;9(9):e102783. doi: 10.1371/journal.pone.0102783.

74. Petty RD, McCarthy NE, Le Dieu R, Kerr JR. MicroRNAs hsa-miR-99b, hsa-miR-330, hsa-miR-126 and hsamiR-30c: Potential Diagnostic Biomarkers in Natural Killer (NK) Cells of Patients with Chronic Fatigue Syndrome (CFS)/ Myalgic Encephalomyelitis (ME). PLoS One. 2016 Mar 11;11(3):e0150904. doi: 10.1371/journal.pone.0150904.

75. Baraniuk JN, Shivapurkar N. Exercise - induced changes in cerebrospinal fluid miRNAs in Gulf War Illness, Chronic Fatigue Syndrome and sedentary control subjects. Sci Rep. 2017 Nov 10;7(1):15338. doi: 10.1038/s41598-017-15383-9. Erratum in: Sci Rep. 2018 Apr 19;8(1):6455.

76. Cording M, Derry S, Phillips T, Moore RA, Wiffen PJ. Milnacipran for pain in fibromyalgia in adults. Cochrane Database Syst Rev. 2015 Oct 20;(10):CD008244. doi: 10.1002/14651858.CD008244.pub3

77. Lunn MP, Hughes RA, Wiffen PJ. Duloxetine for treating painful neuropathy, chronic pain or fibromyalgia. Cochrane Database Syst Rev. 2014 Jan 3;(1):CD007115. doi: 10.1002/14651858.CD007115.pub3. 
78. Walitt B, Urrútia G, Nishishinya MB, Cantrell SE, Häuser W. Selective serotonin reuptake inhibitors for fibromyalgia syndrome. Cochrane Database Syst Rev. 2015 Jun 5;(6):CD011735. doi: 10.1002/14651858.CD011735.

79. Riera R. Selective serotonin reuptake inhibitors for fibromyalgia syndrome. Sao Paulo Med J. 2015 SepOct;133(5):454. doi: 10.1590/1516-3180.20151335T.

80. Moore RA, Derry S, Aldington D, Cole P, Wiffen PJ. Amitriptyline for neuropathic pain in adults. Cochrane Database Syst Rev. 2015 Jul 6;(7):CD008242. doi: 10.1002/14651858.CD008242.pub3.

81. Tort S, Urrútia G, Nishishinya MB, Walitt B. Monoamine oxidase inhibidors (MAOIs) for fibromyalgia syndrome. Cochrane Database Syst Rev. 2012 Apr 18;(4):CD009807. doi: 10.1002/14651858.CD009807.

82. Welsch P, Bernardy K, Derry S, Moore RA, Häuser W. Mirtazapine for fibromyalgia in adults. Cochrane Database Syst Rev. 2018 Aug 6;8:CD012708. doi:10.1002/14651858.CD012708.pub2.

83. Birse F, Derry S, Moore RA. Phenytoin for neuropathic pain and fibromyalgia in adults. Cochrane Database Syst Rev. 2012 May 16;(5):CD009485. doi: 10.1002/14651858.CD009485.

84. Gill D, Derry S, Wiffen PJ, Moore RA. Valproic acid and sodium valproate for neuropathic pain and fibromyalgia in adults. Cochrane Database Syst Rev. 2011 Oct 5;(10):CD009183. doi: 10.1002/14651858.CD009183.pub2.

85. Corrigan R, Derry S, Wiffen PJ, Moore RA. Clonazepam for neuropathic pain and fibromyalgia in adults. Cochrane Database Syst Rev. 2012 May 16;(5):CD009486. doi: 10.1002/14651858.CD009486.pub2.

86. Derry S, Cording M, Wiffen PJ, Law S, Phillips T, Moore RA. Pregabalin for pain in fibromyalgia in adults. Cochrane Database Syst Rev. 2016 Sep 29;9:CD011790. doi: 10.1002/14651858.CD011790.pub2.

87. Wiffen PJ, Derry S, Bell RF, Rice AS, Tölle TR, Phillips T, Moore RA. Gabapentin for chronic neuropathic pain in adults. Cochrane Database Syst Rev. 2017 Jun 9;6:CD007938. doi: 10.1002/14651858.CD007938.pub4.

88. Hearn L, Derry S, Moore RA. Lacosamide for neuropathic pain and fibromyalgia in adults. Cochrane Database Syst Rev. 2012 Feb 15;(2):CD009318. doi: 10.1002/14651858.CD009318.pub2.

89. Wiffen PJ, Derry S, Lunn MP, Moore RA. Topiramate for neuropathic pain and fibromyalgia in adults. Cochrane Database Syst Rev. 2013 Aug 30;(8):CD008314. doi: 10.1002/14651858.CD008314.pub3.

90. Walitt B, Klose P, Üçeyler N, Phillips T, Häuser W. Antipsychotics for fibromyalgia in adults. Cochrane Database Syst Rev. 2016 Jun 2;(6):CD011804. doi: 10.1002/14651858.CD011804.pub2.

91. Walitt B, Klose P, Fitzcharles MA, Phillips T, Häuser W. Cannabinoids for fibromyalgia. Cochrane Database Syst Rev. 2016 Jul 18;7:CD011694. doi: 10.1002/14651858.CD011694.pub2. R

92. Derry S, Wiffen PJ, Häuser W, Mücke M, Tölle TR, Bell RF, Moore RA. Oral nonsteroidal anti-inflammatory drugs for fibromyalgia in adults. Cochrane Database Syst Rev. 2017 Mar 27;3:CD012332. doi: 10.1002/14651858.CD012332.pub2.

93. Gaskell H, Moore RA, Derry S, Stannard C. Oxycodone for pain in fibromyalgia in adults. Cochrane Database Syst Rev. 2016 Sep 1;9:CD012329. doi:10.1002/14651858.CD012329.

94. Larun L, Brurberg KG, Odgaard-Jensen J, Price JR. Exercise therapy for chronic fatigue syndrome. Cochrane Database of Systematic Reviews 2017, Issue 4. Art. No.: CD003200. DOI: 10.1002/14651858.CD003200.pub7.

95. Price JR, Mitchell E, Tidy E, Hunot V. Cognitive behaviour therapy for chronic fatigue syndrome in adults. Cochrane Database of Systematic Reviews 2008, Issue 3. Art. No.: CD001027. DOI: 10.1002/14651858.CD001027.pub2.

96. Adams D, Wu T, Yang X, Tai S, Vohra S. Traditional Chinese medicinal herbs for the treatment of idiopathic chronic fatigue and chronic fatigue syndrome. Cochrane Database of Systematic Reviews 2018, Issue 10. Art. No.: CD006348. DOI: 10.1002/14651858.CD006348.pub3.

97. Collatz A, Johnston SC, Staines DR, Marshall-Gradisnik SM. A Systematic Review of Drug Therapies for Chronic Fatigue Syndrome/Myalgic Encephalomyelitis. Clin Ther. 2016 Jun;38(6):1263-1271.e9. doi: 10.1016/j.clinthera.2016.04.038.

98. Smith ME, Haney E, McDonagh M, Pappas M, Daeges M, Wasson N, et al. Treatment of Myalgic Encephalomyelitis/Chronic Fatigue Syndrome: A Systematic Review for a National Institutes of Health Pathways to Prevention Workshop. Ann Intern Med. 2015 Jun 16;162(12):841-50. doi: 10.7326/M15-0114.

99. Blockmans D, Persoons P. Long-term methylphenidate intake in chronic fatigue syndrome. Acta Clin Belg. 2016 Dec; 71(6):407-414

100. Nilsson MKL, Zachrisson O, Gottfries CG, Matousek M, Peilot B, Forsmark S,Schuit RC, Carlsson ML, Kloberg A, Carlsson A. A randomised controlled trial of the monoaminergic 
stabiliser (-)-OSU6162 in treatment of myalgic encephalomyelitis/chronic fatigue syndrome. Acta Neuropsychiatr. 2018 Jun; 30(3):148-157. doi: 10.1017/neu.2017.3544

101. Proal AD, Albert PJ, Marshall TG, Blaney GP, Lindseth IA. Immunostimulation in the treatment for chronic fatigue syndrome/myalgic encephalomyelitis. Immunol Res. 2013 Jul; 56(2-3):398412. doi: 10.1007/s12026-013-8413-z

102. Plioplys AV, Plioplys S. Amantadine and L-carnitine treatment of Chronic Fatigue Syndrome. Neuropsychobiology. 1997; 35(1):16-23

103. Castro-Marrero J, Cordero MD, Segundo MJ, Sáez-Francàs N, Calvo N, Román-Malo L, Aliste L, Fernández de Sevilla T, Alegre J. Does oral coenzyme Q10 plus NADH supplementation improve fatigue and biochemical parameters in chronic fatigue syndrome? Antioxid Redox Signal. 2015 Mar 10; 22(8):679-85. doi:10.1089/ars.2014.6181

104. Blockmans D, Persoons P, Van Houdenhove B, Lejeune M, Bobbaers H. Combination therapy with hydrocortisone and fludrocortisone does not improve symptoms in chronic fatigue syndrome: a randomized, placebo-controlled, double-blind, crossover study. Am J Med. 2003 Jun 15;114(9):736-41

105. Strayer DR, Carter WA, Stouch BC, Stevens SR, Bateman L, Cimoch PJ, Lapp CW, Peterson DL; Chronic Fatigue Syndrome AMP-516 Study Group, Mitchell WM. A double-blind, placebocontrolled, randomized, clinical trial of the TLR-3 agonist rintatolimod in severe cases of chronic fatigue syndrome. PLoSOne.2012;7(3):e31334. doi:10.1371/journal.pone.0031334

106. Comhaire F. Treating patients suffering from myalgic encephalopathy/chronic fatigue syndrome (ME/CFS) with sodium dichloroacetate: An open-label,proof-of-principle pilot trial. Med Hypotheses. 2018 May; 114:45-48. doi:10.1016/j.mehy.2018.03.002

107. Ostojic SM, Stojanovic M, Drid P, Hoffman JR, Sekulic D, Zenic N. Supplementation with Guanidinoacetic Acid in Women with Chronic Fatigue Syndrome. Nutrients. 2016 Jan 29;8(2):72. doi: 10.3390/nu8020072

108. Gil-Zamorano J, Martin R, Daimiel L, Richardson K, Giordano E, Nicod N, et al. Docosahexaenoic acid modulates the enterocyte Caco-2 cell expression of microRNAs involved in lipid metabolism. J Nutr. 2014 May;144(5):575-85. doi: 10.3945/jn.113.189050.

109. Rodrigues AC, Li X, Radecki L, Pan YZ, Winter JC, Huang M, Yu AM. MicroRNA expression is differentially altered by xenobiotic drugs in different human cell lines. Biopharm Drug Dispos. 2011 Sep;32(6):355-67. doi: 10.1002/bdd.764.

110. Rainer J, Ploner C, Jesacher S, Ploner A, Eduardoff M, Mansha M, et al. Glucocorticoid-regulated microRNAs and mirtrons in acute lymphoblastic leukemia. Leukemia. 2009 Apr;23(4):746-52. doi: 10.1038/leu.2008.370.

111. Lu S, Mukkada VA, Mangray S, Cleveland K, Shillingford N, Schorl C, et al. MicroRNA profiling in mucosal biopsies of eosinophilic esophagitis patients pre and post treatment with steroids and relationship with mRNA targets. PLoS One. 2012;7(7):e40676. doi: 10.1371/journal.pone.0040676.

112. Dave RS, Khalili K. Morphine treatment of human monocyte-derived macrophages induces differential miRNA and protein expression: impact on inflammation and oxidative stress in the central nervous system. J Cell Biochem. 2010 Jul 1;110(4):834-45. doi: 10.1002/jcb.22592.

113. Fayyad-Kazan H, Rouas R, Merimi M, El Zein N, Lewalle P, Jebbawi F, et al. Valproate treatment of human cord blood CD4-positive effector $\mathrm{T}$ cells confers on them the molecular profile (microRNA signature and FOXP3 expression) of natural regulatory CD4-positive cells through inhibition of histone deacetylase. J Biol Chem. 2010 Jul 2;285(27):20481-91. doi: 10.1074/jbc.M110.119628

114. Lukkahatai N, Walitt B, Deandrés-Galiana EJ, Fernández-Martínez JL, Saligan LN. A predictive algorithm to identify genes that discriminate individuals with fibromyalgia syndrome diagnosis from healthy controls. J Pain Res. 2018 Nov 21;11:2981-2990. doi: 10.2147/JPR.S169499. eCollection 2018.

115. Ameling S, Kacprowski T, Chilukoti RK, Malsch C, Liebscher V, Suhre K, et al. Associations of circulating plasma microRNAs with age, body mass index and sex in a population-based study. BMC Med Genomics. 2015 Oct 14;8:61. doi:10.1186/s12920-015-0136-7.

116. Peng X, Li W, Yuan L, Mehta RG, Kopelovich L, McCormick DL. Inhibition of proliferation and induction of autophagy by atorvastatin in PC3 prostate cancer cells correlate with downregulation of Bcl2 and upregulation of miR-182 and p21. PLoS One. 2013 Aug 1;8(8):e70442. doi: 10.1371/journal.pone.0070442. 
117. Minami Y, Satoh M, Maesawa C, Takahashi Y, Tabuchi T, Itoh T, et al. Effect of atorvastatin on microRNA 221 / 222 expression in endothelial progenitor cells obtained from patients with coronary artery disease. Eur J Clin Invest. 2009 May;39(5):359-67. doi: 10.1111/j.1365-2362.2009.02110.x.

118. Saito Y, Suzuki H, Imaeda H, Matsuzaki J, Hirata K, Tsugawa H, et al. The tumor suppressor microRNA$29 \mathrm{c}$ is downregulated and restored by celecoxib in human gastric cancer cells. Int J Cancer. 2013 Apr 15;132(8):1751-60. doi: 10.1002/ijc.27862

119. Li Y, Kong D, Ahmad A, Bao B, Dyson G, Sarkar FH. Epigenetic deregulation of miR-29a and miR-1256 by isoflavone contributes to the inhibition of prostate cancer cell growth and invasion. Epigenetics. 2012 Aug;7(8):940-9. doi: 10.4161/epi.21236.

120. Li Y, Kong D, Ahmad A, Bao B, Sarkar FH. Targeting bone remodeling by isoflavone and 3,3'diindolylmethane in the context of prostate cancer bone metastasis. PLoS One. 2012 Mar;7(3):e33011. doi: 10.1371/journal.pone.0033011.

121. Bao B, Wang Z, Ali S, Ahmad A, Azmi AS, Sarkar SH, et al. Metformin inhibits cell proliferation, migration and invasion by attenuating CSC function mediated by deregulating miRNAs in pancreatic cancer cells. Cancer Prev Res (Phila). 2012 Mar;5(3):355-64. doi: 10.1158/1940-6207.CAPR-11-0299.

122. Chandrasekar V, Dreyer JL. microRNAs miR-124, let-7d and miR-181a regulate cocaine-induced plasticity. Mol Cell Neurosci. 2009 Dec;42(4):350-62. Doi: 10.1016/j.mcn.2009.08.009.

123. Mantri CK, Mantri JV, Pandhare J, Dash C. Methamphetamine inhibits HIV-1 replication in CD4+ T cells by modulating anti-HIV-1 miRNA expression. Am J Pathol. 2014 Jan;184(1):92-100. doi: 10.1016/j.ajpath.2013.09.011.

124. Guo Y, Chen Y, Carreon S, Qiang M. Chronic intermittent ethanol exposure and its removal induce a different miRNA expression pattern in primary cortical neuronal cultures. Alcohol Clin Exp Res. 2012 Jun;36(6):1058-66. doi: 10.1111/j.1530-0277.2011.01689.x.

125. Shin VY, Jin H, Ng EK, Cheng AS, Chong WW, Wong CY, et al. NF- $\kappa B$ targets miR-16 and miR-21 in gastric cancer: involvement of prostaglandin E receptors. Carcinogenesis. 2011 Feb;32(2):240-5. doi: 10.1093/carcin/bgq240.

126. Zhang Y, Pan T, Zhong X, Cheng C. Nicotine upregulates microRNA-21 and promotes TGF- $\beta$-dependent epithelial-mesenchymal transition of esophageal càncer cells. Tumour Biol. 2014 Jul;35(7):7063-72. doi: 10.1007/s13277-014-1968-z.

127. Maccani MA, Avissar-Whiting M, Banister CE, McGonnigal B, Padbury JF, Marsit CJ. Maternal cigarette smoking during pregnancy is associated with downregulation of miR-16, miR-21, and miR-146a in the placenta. Epigenetics. 2010 Oct 1;5(7):583-9.

128. Bhat-Nakshatri P, Wang G, Collins NR, Thomson MJ, Geistlinger TR, Carroll JS, et al. Estradiol-regulated microRNAs control estradiol response in breast cancer cells. Nucleic Acids Res. 2009 Aug;37(14):4850-61. doi: 10.1093/nar/gkp500.

129. Tilghman SL, Bratton MR, Segar HC, Martin EC, Rhodes LV, Li M, et al. Endocrine disruptor regulation of microRNA expression in breast carcinoma cells. PLoS One. 2012;7(3):e32754. doi: 10.1371/journal.pone.0032754

130. Wickramasinghe NS, Manavalan TT, Dougherty SM, Riggs KA, Li Y, Klinge CM. Estradiol downregulates miR-21 expression and increases miR-21 target gene expression in MCF-7 breast cancer cells. Nucleic Acids Res. 2009 May;37(8):2584-95. doi: 10.1093/nar/gkp117.

131. Waltering KK, Porkka KP, Jalava SE, Urbanucci A, Kohonen PJ, Latonen LM, et al. Androgen regulation of micro-RNAs in prostate cancer. Prostate. 2011 May;71(6):604-14. doi: 10.1002/pros.21276.

132. Yu X, Zhang X, Dhakal IB, Beggs M, Kadlubar S, Luo D. Induction of cell proliferation and survival genes by estradiol-repressed microRNAs in breast cancer cells. BMC Cancer. 2012 Jan 20;12:29. doi: 10.1186/14712407-12-29.

133. Feng B, Cao Y, Chen S, Ruiz M, Chakrabarti S. miRNA-1 regulates endothelin-1 in diabetes. Life Sci. 2014 Mar 7;98(1):18-23. doi: 10.1016/j.lfs.2013.12.199.

134. Du B, Ma LM, Huang MB, Zhou H, Huang HL, Shao P, Chen YQ, Qu LH. High glucose down-regulates miR-29a to increase collagen IV production in HK-2 cells. FEBS Lett. 2010 Feb 19;584(4):811-6. doi: 10.1016/j.febslet.2009.12.053.

135. Bae J, Won M, Kim DY, Kim JH, Kim YM, Kim YT, et al. Identification of differentially expressed microRNAs in endometrial cancer cells after progesterone treatment. Int J Gynecol Cancer. 2012 May;22(4):561-5. doi:10.1097/IGC.0b013e31824927db. 
136. Cochrane DR, Jacobsen BM, Connaghan KD, Howe EN, Bain DL, Richer JK. Progestin regulated miRNAs that mediate progesterone receptor action in breast cancer. Mol Cell Endocrinol. 2012 May 15;355(1):1524. doi: 10.1016/j.mce.2011.12.020.

137. Wang WL, Chatterjee N, Chittur SV, Welsh J, Tenniswood MP. Effects of $1 \alpha, 25$ dihydroxyvitamin D3 and testosterone on miRNA and mRNA expression in LNCaP cells. Mol Cancer. 2011 May 18;10:58. doi: 10.1186/1476-4598-10-58.

138. Jorde R, Svartberg J, Joakimsen RM, Coucheron DH. Plasma profile of microRNA after supplementation with high doses of vitamin D3 for 12 months. BMC Res Notes. 2012 May 17;5:245. doi: 10.1186/1756-05005-245.

139. Chang CM, Warren JL, Engels EA. Chronic fatigue syndrome and subsequent risk of cancer among elderly US adults. Cancer. 2012 Dec 1;118(23):5929-36. doi:10.1002/cncr.27612.

140. Daniels SI, Sillé FC, Goldbaum A, Yee B, Key EF, Zhang L, et al. Improving power to detect changes in blood miRNA expression by accounting for sources of variability in experimental designs. Cancer Epidemiol Biomarkers Prev. 2014 Dec;23(12):2658-66. doi: 10.1158/1055-9965.EPI-14-0623.

141. Foye C, Yan IK, David W, Shukla N, Habboush Y, Chase L, et al. Comparison of miRNA quantitation by Nanostring in serum and plasma samples. PLoS One. 2017 Dec 6;12(12):e0189165. doi: 10.1371/journal.pone.0189165.

142. Ludwig N, Leidinger P, Becker K, Backes C, Fehlmann T, Pallasch C, et al. Distribution of miRNA expression across human tissues. Nucleic Acids Res. 2016 May 5;44(8):3865-77. doi: 10.1093/nar/gkw116.

143. Awan HM, Shah A, Rashid F, Shan G. Primate-specific Long Non-coding RNAs and MicroRNAs. Genomics Proteomics Bioinformatics. 2017 Jun;15(3):187-195. doi:10.1016/j.gpb.2017.04.002. 